\title{
Search for heavy resonances that decay into a vector boson and a Higgs boson in hadronic final states at $\sqrt{s}=13 \mathrm{TeV}$
}

\author{
CMS Collaboration* \\ CERN, 1211 Geneva 23, Switzerland
}

Received: 5 July 2017 / Accepted: 1 September 2017 / Published online: 22 September 2017

(C) CERN for the benefit of the CMS collaboration 2017. This article is an open access publication

\begin{abstract}
A search for heavy resonances with masses above $1 \mathrm{TeV}$, decaying to final states containing a vector boson and Higgs boson, is presented. The search considers hadronic decays of the vector boson, and Higgs boson decays to b quarks. The decay products are highly boosted, and each collimated pair of quarks is reconstructed as a single, massive jet. The analysis is performed using a data sample collected in 2016 by the CMS experiment at the LHC in proton-proton collisions at a center-of-mass energy of $13 \mathrm{TeV}$, corresponding to an integrated luminosity of $35.9 \mathrm{fb}^{-1}$. The data are consistent with the background expectation and are used to place limits on the parameters of a theoretical model with a heavy vector triplet. In the benchmark scenario with massdegenerate $\mathrm{W}^{\prime}$ and $\mathrm{Z}^{\prime}$ bosons decaying predominantly to pairs of standard model bosons, for the first time heavy resonances for masses as high as $3.3 \mathrm{TeV}$ are excluded at $95 \%$ confidence level, setting the most stringent constraints to date on such states decaying into a vector boson and a Higgs boson.
\end{abstract}

\section{Introduction}

The discovery of the Higgs boson $(\mathrm{H})$ at the CERN LHC [1$3]$ represents a milestone in the understanding of the standard model (SM) of particle physics. However, the degree of fine-tuning required to accommodate the observed mass of $125 \mathrm{GeV}$ [4-7] suggests the presence above $1 \mathrm{TeV}$ of new heavy particles beyond the SM (BSM), possibly lying within reach of the LHC. These resonances, denoted as X, are expected to be connected to the electroweak sector of the SM, with significant couplings to the SM bosons. Hence, these heavy resonances potentially could be observed through their decay into a vector boson $(\mathrm{V}=\mathrm{W}$ or $\mathrm{Z})$ and a Higgs boson.

\footnotetext{
^e-mail: cms-publication-committee-chair@cern.ch
}

The $\mathrm{VH}$ resonances are predicted in several BSM theoretical models, most notably weakly coupled spin-1 $Z^{\prime}[8,9]$ and $\mathrm{W}^{\prime}$ models [10], strongly coupled composite Higgs models [11-13], and little Higgs models [14-16]. The heavy vector triplet (HVT) framework [17] extends the SM by introducing a triplet of heavy vector bosons, one neutral $\mathrm{Z}^{\prime}$ and two charged $\mathrm{W}^{\prime} \mathrm{s}$, collectively represented as $\mathrm{V}$ ' and degenerate in mass. The heavy vector bosons couple to SM bosons and fermions with strengths $g_{\mathrm{V}} c_{\mathrm{H}}$ and $g^{2} c_{\mathrm{F}} / g_{\mathrm{V}}$, respectively, where $g_{\mathrm{V}}$ is the strength of the new interaction, $c_{\mathrm{H}}$ is the coupling between the HVT bosons, the Higgs boson, and longitudinally polarized SM vector bosons, $c_{\mathrm{F}}$ is the coupling between the HVT bosons and the SM fermions, and $g$ is the $S U(2)_{L}$ gauge coupling. In this paper, two different benchmark scenarios are considered [17]. In model A $\left(g_{\mathrm{V}}=1\right.$, $\left.c_{\mathrm{H}}=-0.556, c_{\mathrm{F}}=-1.316\right)$, the coupling strengths to the SM bosons and fermions are comparable, and the new particles decay primarily to fermions. In model $\mathrm{B}\left(g_{\mathrm{V}}=3\right.$, $\left.c_{\mathrm{H}}=-0.976, c_{\mathrm{F}}=1.024\right)$, the couplings to fermions are suppressed with respect to the couplings to bosons, resulting in a branching fraction to SM bosons close to unity.

This paper describes the search in proton-proton collisions at $13 \mathrm{TeV}$ for heavy resonances decaying to final states containing a SM vector boson and a Higgs boson, which subsequently decay into a pair of quarks and a pair of b quarks, respectively. Use of the hadronic decay modes takes advantage of the large branching fractions, which compensate for the effect of the large multijet background. This search concentrates on the high mass region, as previous searches [1825] have excluded $m_{X}$ in the region below a few TeV. As a result of the large resonance mass, the two bosons produced in the decay have large Lorentz boosts in the laboratory frame, and consequently the hadronic decay products of each boson tend to be clustered within a single hadronic jet. The jet mass, substructure, and b tagging information are crucial to identifying hadronically decaying vector bosons and Higgs boson candidates, and to discriminating against the dominant SM backgrounds. 
This search complements and significantly extends the reach of the CMS search with 2015 data for $\mathrm{VH}$ resonances with semileptonic decay modes of the vector bosons [24], which excludes at $95 \%$ confidence level (CL) $\mathrm{W}^{\prime}$ and $\mathrm{Z}^{\prime}$ resonances with mass below $1.6 \mathrm{TeV}$ and mass-degenerate $\mathrm{V}$ ' resonances with masses up to $2.0 \mathrm{TeV}$ in the HVT benchmark model B. The ATLAS Collaboration has performed a search in the same final state with a comparable data set, excluding $\mathrm{W}^{\prime}$ and $\mathrm{Z}^{\prime}$ bosons with masses below 2.2 and $1.6 \mathrm{TeV}$, respectively, and a V' boson with mass below $2.3 \mathrm{TeV}$ in the HVT model B scenario [25].

\section{Data and simulated samples}

The data sample studied in this analysis was collected in 2016 with the CMS detector in proton-proton collisions at a center-of-mass energy of $13 \mathrm{TeV}$, and corresponds to an integrated luminosity of $35.9 \mathrm{fb}^{-1}$.

Simulated signal events are generated at leading order (LO) with the MADGRAPH5_aMC@NLO 2.2.2 matrix element generator [26]. The Higgs boson is required to decay into a $\mathrm{b} \overline{\mathrm{b}}$ pair, and the vector boson to decay hadronically. Other decay modes are not considered in the present analysis. Different hypotheses for the heavy resonance mass $m_{X}$ in the range 1000 to $4500 \mathrm{GeV}$ are considered, assuming a narrow resonance width $(0.1 \%$ of the mass), which is small with respect to the experimental resolution. This narrowwidth assumption is valid in a large fraction of the HVT parameter space, and fulfilled in both benchmark models A and B [17].

Although the background is estimated using a method based on data, simulated background samples are generated for the optimization of the analysis selections. Multijet background events are generated at LO with MADGRAPH5_aMC@NLO, and top quark pair production is simulated at next-to-leading order (NLO) with the POWHEG 2.0 generator [27-29] and rescaled to the cross section computed with ToP++ v2.0 [30] at next-to-next-to-leading order. Other SM backgrounds, such as $\mathrm{W}+$ jets, $\mathrm{Z}+\mathrm{jets}$, single top quark production, VV, and nonresonant VH production, are simulated at NLO in QCD with MADGRAPH5_aMC@NLO using the FxFx merging scheme [31]. Parton showering and hadronization processes are interfaced with PYTHIA 8.205 [32] with the CUETP8M1 underlying event tune [33, 34]. The CUETP8M2T4 tune [35] is used for top quark pair production. The NNPDF 3.0 [36] parton distribution functions (PDFs) are used in generating all simulated samples. Additional collisions in the same or adjacent bunch crossings (pileup) are taken into account by superimposing simulated minimum bias interactions onto the hard scattering process, with a frequency distribution matching that observed experimentally. The generated events are processed through a full detector simulation based on GEANT4 [37] and reconstructed with the same algorithms as used for collision data.

\section{The CMS detector}

The central feature of the CMS detector is a superconducting solenoid with a $6 \mathrm{~m}$ internal diameter. In the solenoid volume, a silicon pixel and strip tracker measures charged particles within the pseudorapidity range $|\eta|<2.5$. The tracker consists of 1440 silicon pixel and 15,148 silicon strip detector modules and is located in the $3.8 \mathrm{~T}$ field of the solenoid. For nonisolated particles of transverse momentum $1<p_{\mathrm{T}}<10 \mathrm{GeV}$ and $|\eta|<1.4$, the track resolutions are typically $1.5 \%$ in $p_{\mathrm{T}}$ and $25-90(45-150) \mu \mathrm{m}$ in the transverse (longitudinal) impact parameter [38]. A lead tungstate crystal electromagnetic calorimeter (ECAL), and a brass and scintillator hadron calorimeter (HCAL), each composed of a barrel and two endcap sections, provide coverage up to $|\eta|<3.0$, which is further extended by forward calorimeters. Muons are measured in drift tubes, cathode strip chambers, and resistive-plate chambers embedded in the steel fluxreturn yoke outside the solenoid.

The first level of the CMS trigger system [39], composed of custom hardware processors, uses information from the calorimeters and muon detectors to select the most interesting events in a fixed time interval of less than $4 \mu \mathrm{s}$. The highlevel trigger (HLT) processor farm decreases the event rate from around $100 \mathrm{kHz}$ to about $1 \mathrm{kHz}$, before data storage.

A detailed description of the CMS detector, together with a definition of the coordinate system used and the relevant kinematic variables, can be found in Ref. [40].

\section{Event reconstruction}

The event reconstruction employs a particle-flow (PF) algorithm $[41,42]$, which uses an optimized combination of information from the various elements of the CMS detector to reconstruct and identify individual particles produced in each collision. The algorithm identifies each reconstructed particle either as an electron, a muon, a photon, a charged hadron, or a neutral hadron. The PF candidates are clustered into jets using the anti- $k_{\mathrm{T}}$ algorithm $[43,44]$ with a distance parameter $R=0.8$, after passing the charged-hadron subtraction (CHS) pileup mitigation algorithm [45]. For each event, a primary vertex is identified as the one with the highest sum of the $p_{\mathrm{T}}^{2}$ of the associated reconstructed objects, jets and identified leptons, and missing transverse momentum. The CHS algorithm removes charged PF candidates with a track longitudinal impact parameter not compatible with this primary vertex. The contribution to a jet of neutral particles originating from pileup interactions, assumed to be proportional to 
the jet area [46], is subtracted from the jet energy. Jet energy corrections as a function of the $p_{\mathrm{T}}$ and $\eta$ are extracted from simulation and data in dijet, multijet, $\gamma+$ jets, and leptonic $\mathrm{Z}+$ jets events. The jet energy resolution typically amounts to $5 \%$ at $1 \mathrm{TeV}[47,48]$. Jets are required to pass identification criteria in order to remove spurious jets arising from detector noise [49]. This requirement has negligible impact on the signal efficiency.

Although AK8 CHS jets are considered for their kinematic properties, the mass of the jet and the substructure variables are determined with a more sophisticated algorithm than the CHS procedure, denoted as pileup-per-particle identification (PUPPI) [50]. The PUPPI algorithm uses a combination of the three-momenta of the particles, event pileup properties, and tracking information in order to compute a weight, assigned to charged and neutral candidates, describing the likelihood that each particle originates from a pileup interaction. The weight is used to rescale the particle four-momenta, superseding the need for further jet-based corrections. The PUPPI constituents are subsequently clustered with the same algorithm used for CHS jets, and then are matched with near $100 \%$ efficiency to the AK8 jets clustered with the CHS constituents.

The soft-drop algorithm [51,52], which is designed to remove contributions from soft radiation and additional interactions, is applied to PUPPI jets. The angular exponent parameter of the algorithm is set to $\beta=0$, and the soft threshold to $z_{\text {cut }}=0.1$. The soft-drop jet mass is defined as the invariant mass associated with the four-momentum of the jet after the application of the soft-drop algorithm. Dedicated mass corrections, derived from simulation and data in a region enriched with $t \bar{t}$ events having merged $\mathrm{W}(\mathrm{q} \overline{\mathrm{q}})$ decays, are applied to each jet mass in order to remove any residual jet $p_{\mathrm{T}}$ dependence [53], and to match the jet mass scale and resolution observed in data. The measured jet mass resolution, obtained after applying the PUPPI and soft-drop algorithms, is approximately $10 \%$.

Substructure variables are used to identify single reconstructed jets that result from the merger of more than one parton jet. These variables are calculated on each reconstructed jet before the application of the soft-drop algorithm including the PUPPI algorithm corrections for pileup mitigation. The constituents of the jet are clustered iteratively with the anti- $k_{\mathrm{T}}$ algorithm, and the procedure is stopped when $N$ subjets are obtained. A variable, the $N$-subjettiness [54], is introduced:

$\tau_{N}=\frac{1}{d_{0}} \sum_{k} p_{\mathrm{T}, k} \min \left(\Delta R_{1, k}, \Delta R_{2, k}, \ldots, \Delta R_{N, k}\right)$.

The index $k$ runs over the jet constituents and the distances $\Delta R_{J, k}$ are calculated with respect to the axis of the $J$ th subjet. The normalization factor $d_{0}$ is calculated as $d_{0}=$ $\sum_{k} p_{\mathrm{T}, k} R_{0}$, setting $R_{0}$ to the radius of the original jet. The variable that best discriminates between quark and gluon jets and jets from two-body decays of massive particles is the ratio of 2-subjettiness and 1-subjettiness, $\tau_{21}=\tau_{2} / \tau_{1}$, which lies in the interval from 0 to 1 , where small values correspond to a high compatibility with the hypothesis of a massive object decaying into two quarks. The normalization scale factors relative to the $\tau_{21}$ categories are measured from data in a sample enriched in $\mathrm{t} \overline{\mathrm{t}}$ events in two $\tau_{21}$ intervals $(0.99 \pm 0.11$ for $\tau_{21}<0.35$, and $1.03 \pm 0.23$ for $0.35<\tau_{21}<0.75$ ) [53]. These two selections are approximately 50 and $45 \%$ efficient for identifying two-pronged jets produced in a decay of a massive boson, and 10 and $60 \%$ efficient on one-pronged jets, respectively. The threshold values are chosen in order to maximize the overall sensitivity over the entire mass spectrum.

The Higgs boson jet candidates are identified using a dedicated $\mathrm{b}$ tagging discriminator, specifically designed to identify a pair of $b$ quarks clustered in a single jet [55]. The algorithm combines information from displaced tracks and the presence of one or two secondary vertices within the Higgs boson jet in a dedicated multivariate algorithm. The decay chains of the two $b$ hadrons are resolved by associating reconstructed secondary vertices with the directions of the two $N$-subjettiness axes. Tight and loose operating points are chosen for Higgs boson jets that have corresponding falsepositive rates for light quark and gluon jets being identified as jets from $b$ quarks of about 0.8 and $8 \%$, with efficiencies of approximately 35 and $75 \%$, respectively. Scale factors, derived from data in events enriched by jets containing muons [55], are applied to the simulation to correct for the differences between data and simulation.

Since the analysis concentrates on hadronic final states, events containing isolated charged leptons or large missing transverse momentum are rejected. Electrons are reconstructed in the fiducial region $|\eta|<2.5$ by matching the energy deposits in the ECAL with tracks reconstructed in the tracker [56]. Muons are reconstructed within the acceptance of the CMS muon systems, $|\eta|<2.4$, using the information from both the muon spectrometer and the silicon tracker [57]. The isolation of electrons and muons is based on the summed energy of reconstructed PF candidates within a cone around the lepton direction. Hadronically decaying $\tau$ leptons are reconstructed in the $|\eta|<2.3$ region by combining one or three hadronic charged PF candidates with up to two neutral pions, the latter also reconstructed by the $\mathrm{PF}$ algorithm from the photons arising from the $\pi^{0} \rightarrow \gamma \gamma$ decay [58]. The missing transverse momentum is calculated as the magnitude of the vector sum of the momenta of all PF candidates projected onto the plane perpendicular to the beams. 


\section{Event selection}

Events are collected with four triggers [39]. The first requires $H_{\mathrm{T}}$, defined as the scalar sum of the transverse momentum of the PF jets, to be larger than 800 or $900 \mathrm{GeV}$, depending on the instantaneous luminosity. The second trigger, with a lower $H_{\mathrm{T}}$ threshold set to $650 \mathrm{GeV}$, is also required to have a pair of PF jets with invariant mass larger than $950 \mathrm{GeV}$, and pseudorapidity separation $|\Delta \eta|$ smaller than 1.5 . A third trigger requires at least one PF jet with $p_{\mathrm{T}}$ larger than 450 $\mathrm{GeV}$. The fourth trigger selects events with at least one PF jet with $p_{\mathrm{T}}>360 \mathrm{GeV}$ passing a trimmed mass [59] threshold of $30 \mathrm{GeV}$, or $H_{\mathrm{T}}>700 \mathrm{GeV}$ and trimmed mass larger than $50 \mathrm{GeV}$. In all these triggers, reconstruction of PF jets is based on the anti- $k_{\mathrm{T}}$ algorithm with $R=0.4$, rather than $R=0.8$ as used offline.

In the offline preselection, the two jets with highest $p_{\mathrm{T}}$ in the event are required to have $p_{\mathrm{T}}>200 \mathrm{GeV}$ and $|\eta|<2.5$, and $|\Delta \eta| \leq 1.3$. At least one of the two jets must have a soft-drop jet mass compatible with the Higgs boson mass, $105<m_{\mathrm{j}}<135 \mathrm{GeV}$ (H jet), and the other jet a mass compatible with the mass of the vector bosons, $65<m_{\mathrm{j}}<105 \mathrm{GeV}$ ( $\mathrm{V}$ jet). The jet mass categorization is shown in Fig. 1. The $\mathrm{H}$ jet and $\mathrm{V}$ jet candidates are required to have a combined invariant mass $m_{\mathrm{VH}}$ larger than $985 \mathrm{GeV}$, to avoid trigger threshold effects and thus ensure full efficiency. Events with isolated electrons or muons with $p_{\mathrm{T}}>10 \mathrm{GeV}$, or $\tau$ leptons with $p_{\mathrm{T}}>18 \mathrm{GeV}$, are rejected. The reconstructed missing transverse momentum is required to be smaller than $250 \mathrm{GeV}$.

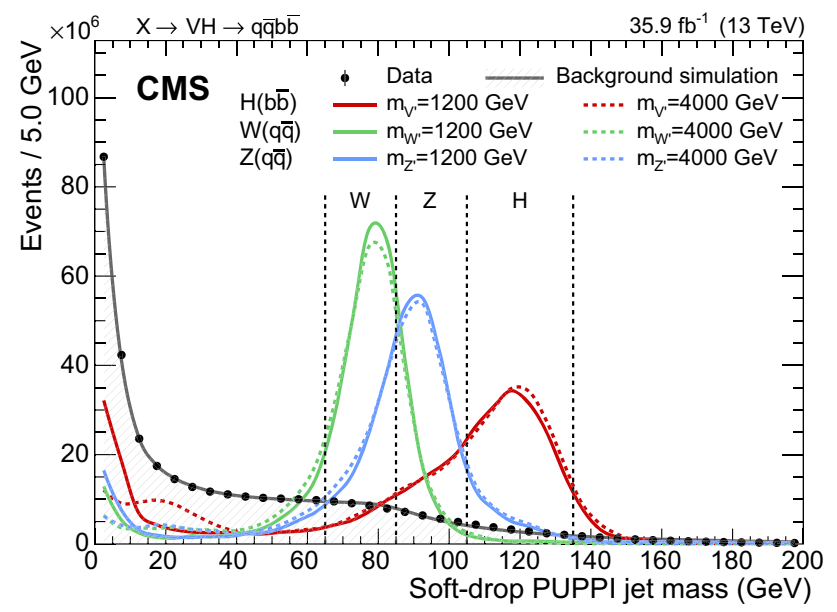

Fig. 1 Distribution of the soft-drop PUPPI mass after the kinematic selections on the two jets, for data, simulated background, and signal. The signal events with low mass correspond to boson decays where one of the two quarks is emitted outside the jet cone or the two quarks are overlapping. The distributions are normalized to the number of events observed in data. The dashed vertical lines represent the boundaries between the jet mass categories
The events passing the preselection are divided into eight exclusive categories. Two categories are defined for the $\mathrm{H}$ jet, depending on the value of the $b$ tagging discriminator: $a$ tight category containing events with a discriminator larger than 0.9 , and a loose category requiring a value between 0.3 and 0.9 . Similarly, two categories of $\mathrm{V}$ jets are defined using the subjettiness ratio: a high purity category containing events with $\tau_{21} \leq 0.35$, and a low purity category having $0.35<\tau_{21}<0.75$. Although it is expected that the tight and high purity categories dominate the total sensitivity, the loose and low purity categories are retained since for large dijet invariant mass they provide a nonnegligible signal efficiency with an acceptable level of background contamination.

Two further categories are defined based on the $\mathrm{V}$ jet mass, by splitting the mass interval. Events with $\mathrm{V}$ jet mass closer to the nominal $\mathrm{W}$ boson mass value, $65<m_{\mathrm{j}} \leq 85 \mathrm{GeV}$, are assigned to a $\mathrm{W}$ mass category, and those with $85<$ $m_{\mathrm{j}} \leq 105 \mathrm{GeV}$ fall into a $\mathrm{Z}$ mass category. Even if the $\mathrm{W}$ and $\mathrm{Z}$ mass peaks cannot be fully resolved, this classification allows a partial discrimination between a potential $\mathrm{W}^{\prime}$ or $\mathrm{Z}^{\prime}$ signal. The signal efficiency for the combination of the eight categories reaches $36 \%$ at $m_{\mathrm{X}}=1.2-1.6 \mathrm{TeV}$, and slowly decreases to $21 \%$ at $m_{\mathrm{X}}=4.5 \mathrm{TeV}$. The $N$-subjettiness and $\mathrm{b}$ tagging categorizations are shown in Fig. 2.

\section{Background estimation}

The background is largely dominated by multijet production, which accounts for more than $95 \%$ of the total background. The top quark pair contribution is approximately $3-4 \%$, depending on the category. The remaining fraction is composed of vector boson production in association with partons, and SM diboson processes.

The background is estimated directly from data, assuming that the $m_{\mathrm{VH}}$ distribution can be described by a smooth, parametrizable, monotonically decreasing function. This assumption is verified in the $\mathrm{V}$ jet mass sidebands $(40<$ $m_{\mathrm{j}}<65 \mathrm{GeV}$ ) and in simulation. The expressions considered are functions of the variable $x=m_{\mathrm{VH}} / \sqrt{s}$, where $\sqrt{s}=13 \mathrm{TeV}$ is the center of mass energy, and the number of parameters $p_{i}$, including the normalization, is between two and five:

$$
\begin{aligned}
& \frac{p_{0}}{x^{p_{1}}}, \quad \frac{p_{0}(1-x)^{p_{1}}}{x^{p_{2}}}, \quad \frac{p_{0}(1-x)^{p_{1}}}{x^{p_{2}+p_{3} \log (x)}}, \\
& \frac{p_{0}(1-x)^{p_{1}}}{x^{p_{2}+p_{3} \log (x)+p_{4} \log ^{2}(x)}} .
\end{aligned}
$$

Starting from the simplest functional form, an iterative procedure based on the Fisher F test [60] is used to check at $10 \%$ $\mathrm{CL}$ if additional parameters are needed to model the background distribution. For most categories, the two-parameter functional form is found to describe the data spectrum suf- 

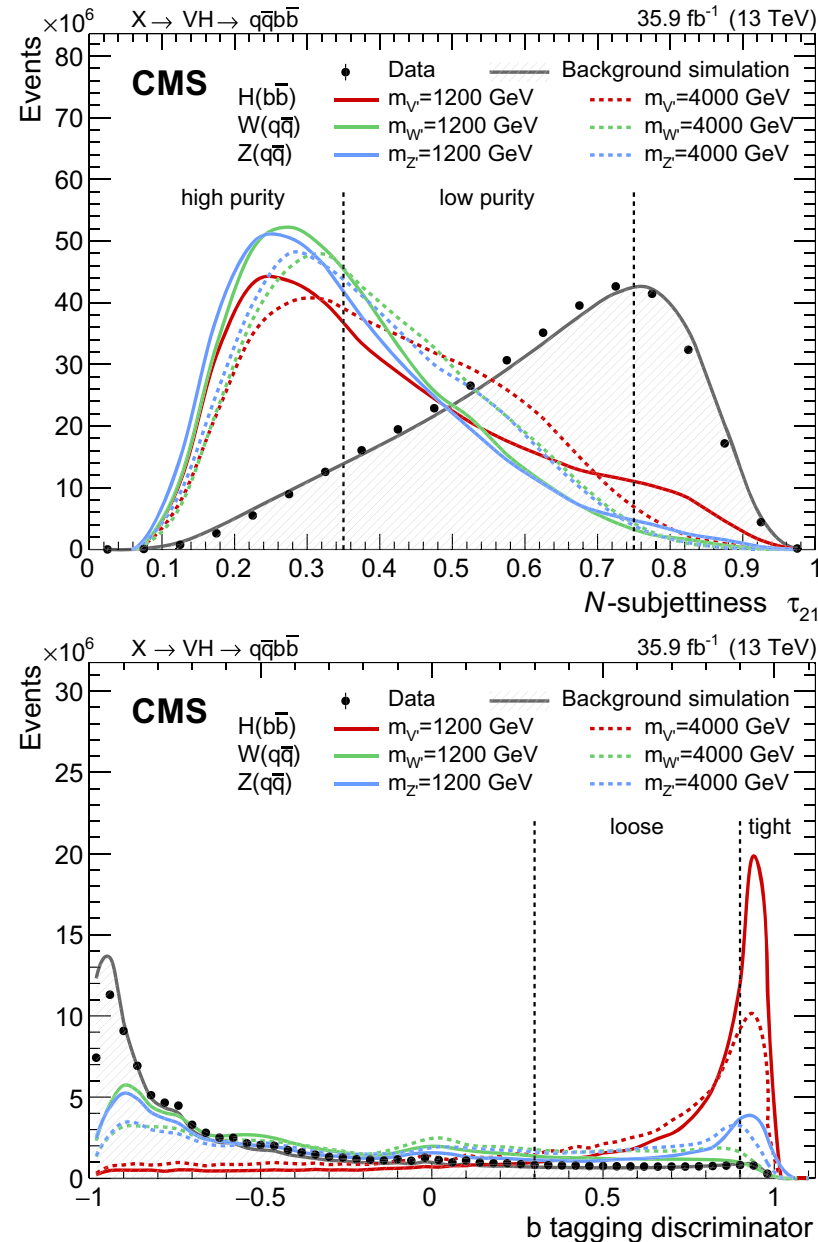

Fig. 2 Distribution of the $N$-subjettiness $\tau_{21}$ (upper) and b tagging discriminator output (lower) after the kinematic selections on the two jets, for data, simulated background, and signal. The distributions are normalized to the number of events observed in data. The dashed vertical lines represent the boundaries between the categories as described in the text

ficiently well. However, in more populated categories, with loose $b$ tagging or low purity, three- or four-parameter functions are preferred. The results of the fits are shown in Figs. 3 and 4 for the $\mathrm{W}$ and $\mathrm{Z}$ mass regions, respectively. Although the fits are unbinned, the binning chosen to present the results is consistent with the detector resolution. The event with the highest invariant mass observed has $m_{\mathrm{VH}}=4920 \mathrm{GeV}$ and is in the $\mathrm{W}$ mass, low purity, tight $\mathrm{b}$ tag category.

The shape of the reconstructed signal mass distribution is extracted from the simulated signal samples. The signal shape is parametrized separately for each channel with a Gaussian peak and a power law to model the lower tail, for a total of four parameters. The reconstruction resolution for $m_{\mathrm{VH}}$ is taken to be the width of the Gaussian core, and is $4 \%$ at low resonance mass and 3\% at high mass.

Dedicated tests have been performed to check the robustness of the fit method by generating pseudo-experiments after injecting a simulated signal with various mass values and cross sections on top of the nominal fitted function. The pseudo-data distribution is then subjected to the same procedure as the data, including the F test, to determine the background function. The signal yield derived from a combined background and signal fit is found to be compatible with the injected yield within one third of the statistical uncertainty, regardless of the injected signal strength and resonance mass. These tests verify that the possible presence of a signal and the choice of the function used to model the background do not introduce significant biases in the final result.

\section{Systematic uncertainties}

The background estimation is obtained from the fit to the data in the considered categories. As such, the only relevant uncertainty originates from the covariance matrix of the dijet function fit, as indicated by the shaded region in Figs. 3 and 4 .

The dominant uncertainties in the signal arise from the $\mathrm{H}$ jet and $\mathrm{V}$ jet tagging. The $\mathrm{b}$ tagging scale factor uncertainties [55] are varied by one standard deviation, and the difference in the signal yield is estimated to be $4-8 \%$ for the tight categories and $2-5 \%$ for the loose categories. The same procedure is applied to the $\tau_{21}$ scale factors, whose uncertainty is measured to be $11 \%$ for the high purity and $23 \%$ for the low purity category, as reported in Sect. 4 . The uncertainties associated with the Higgs boson mass selection and the $\mathrm{V}$ jet tagging extrapolation from the $\mathrm{t} \overline{\mathrm{t}}$ scale to larger jet $p_{\mathrm{T}}$ are estimated by using an alternative HERWIG++ [61] shower model, and are found to be $5-7 \%$ and $3-20 \%$ for the $\mathrm{H}$ and $\mathrm{V}$ jet candidates, respectively. Both $\mathrm{b}$ tagging and $\tau_{21}$ uncertainties are anti-correlated between the corresponding categories.

Uncertainties in the reconstruction of the hadronic jets affect both the signal efficiency and the shape of the reconstructed resonance mass. The four-momenta of the reconstructed jets are scaled and smeared according to the uncertainties in the jet $p_{\mathrm{T}}$ and momentum resolution. These effects account for a $1 \%$ uncertainty in the mean and a $2 \%$ uncertainty in the width of the signal Gaussian core. The jet mass is also scaled and smeared according to the measurement of the jet mass scale (resolution), giving rise to $2 \%(12 \%)$ normalization uncertainties, respectively, and up to $16 \%(18 \%)$ migration effects between the $\mathrm{W}$ and $\mathrm{Z}$ mass regions depending on the category and signal hypothesis.

Additional systematic uncertainties affecting the signal normalization include the lepton identification, isolation and missing transverse momentum vetoes (accounting for $1 \%$ each), pileup modeling $(0.1 \%)$, the integrated luminosity (2.5\%) [62], and the choice of the PDF set [63] (1\% for acceptance, $6-25 \%$ for the normalization). The factorization and 

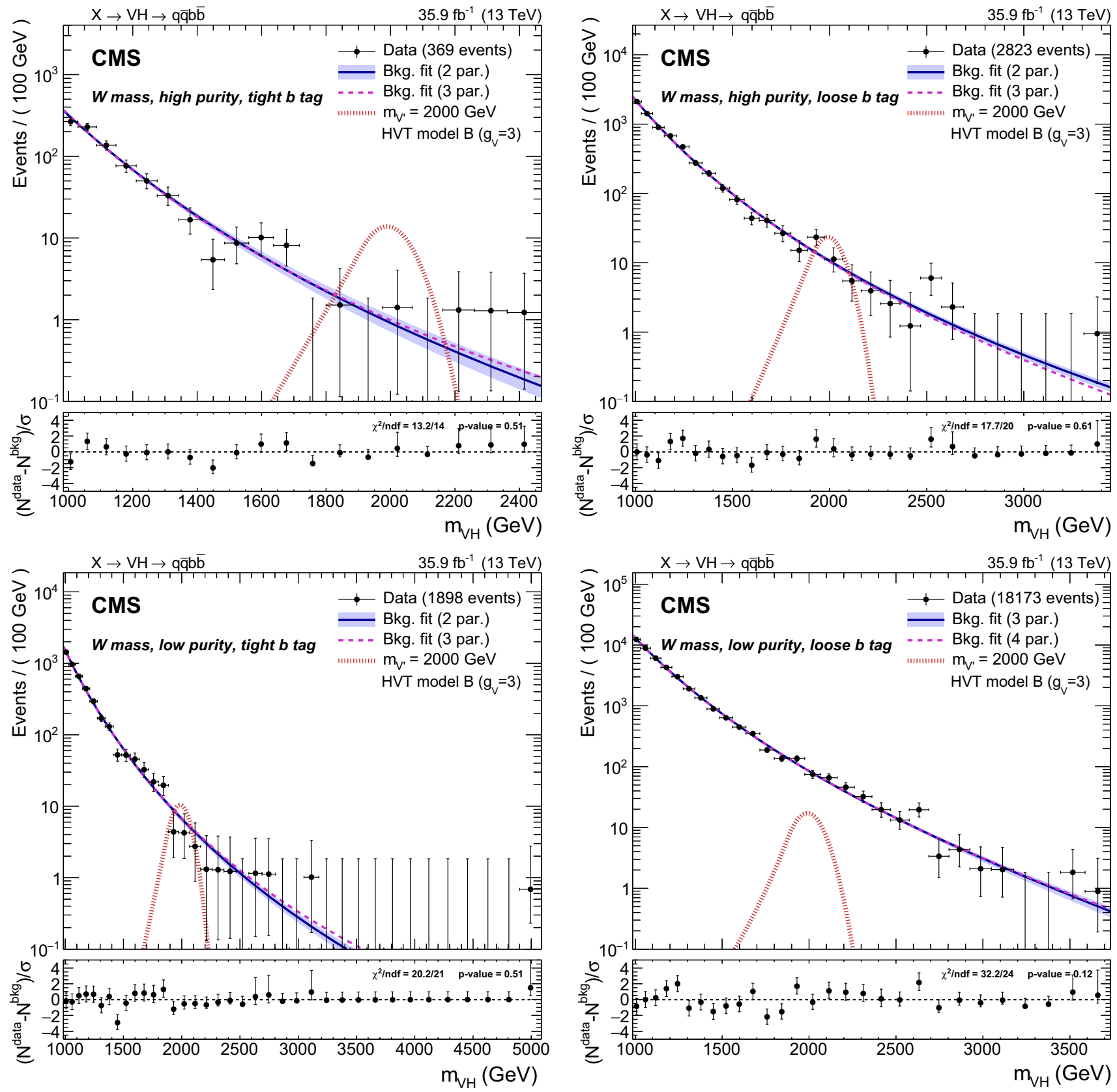

Fig. 3 Dijet invariant distribution $m_{\mathrm{VH}}$ of the two leading jets in the W mass region: high purity (upper) and low purity (lower) categories, with tight (left) and loose (right) b tagging selections. The preferred background-only fit is shown as a solid blue line with an associated shaded band indicating the uncertainty. An alternative fit is shown as a purple dashed line. The ratio panels show the pulls in each bin,

$\left(N^{\text {data }}-N^{\text {bkg }}\right) / \sigma$, where $\sigma$ is the Poisson uncertainty in data. The horizontal bars on the data points indicate the bin width and the vertical bars represent the normalized Poisson errors, and are shown also for bins with zero entries up to the highest $m_{\mathrm{VH}}$ event. The expected contribution of a resonance with $m_{\mathrm{X}}=2000 \mathrm{GeV}$, simulated in the context of the HVT model $\mathrm{B}$, is shown as a dot-dashed red line

renormalization scale uncertainties are estimated by varying the scales up and down by a factor of 2 , and the resulting effect is a variation of $4-13 \%$ in the normalization of the signal events.

\section{Results and interpretation}

Results are obtained by fitting the background functions and the signal shape to the unbinned data $m_{\mathrm{VH}}$ distributions in 

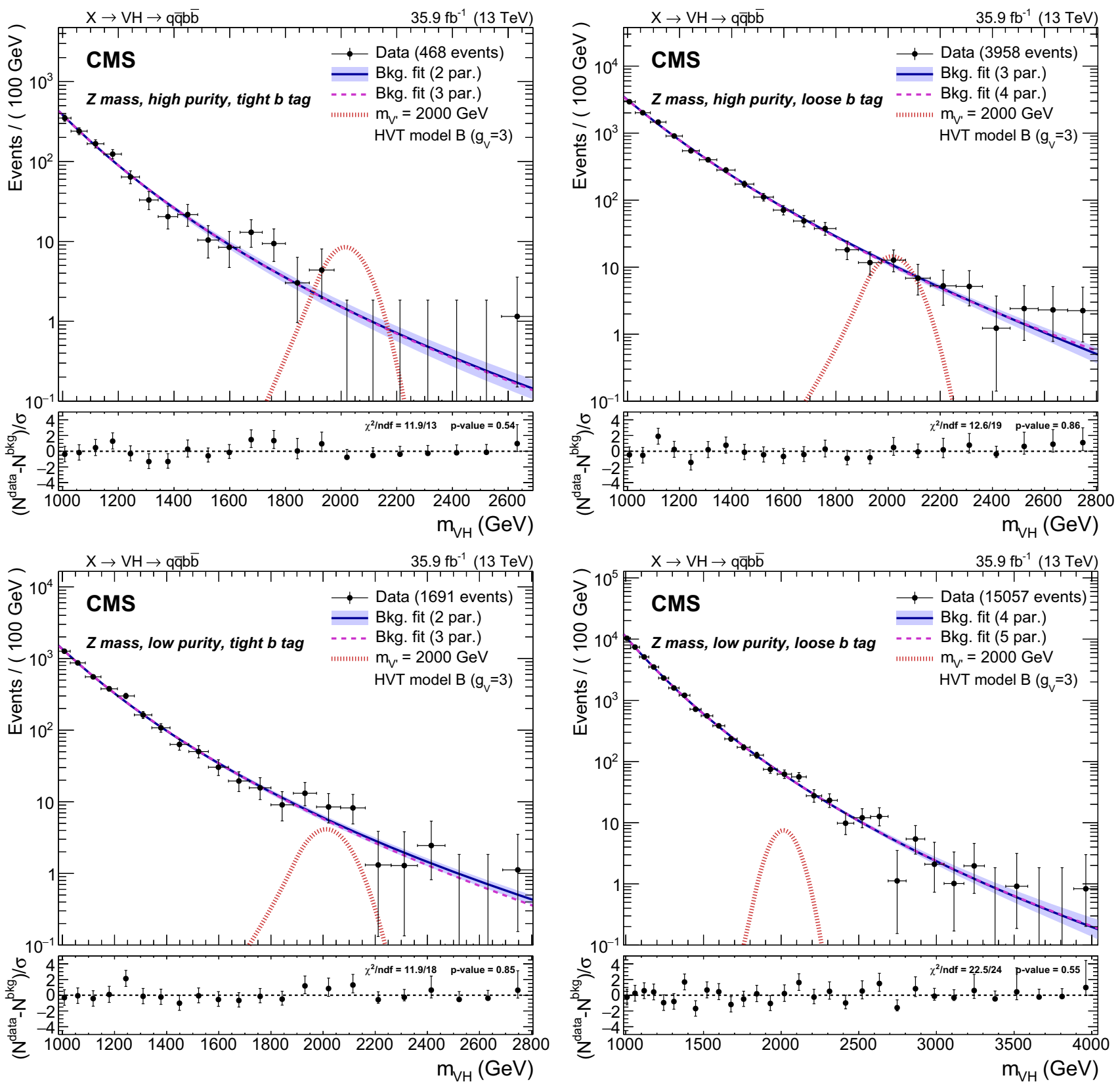

Fig. 4 Dijet invariant distribution $m_{\mathrm{VH}}$ of the two leading jets in the $\mathrm{Z}$ mass region: high purity (upper) and low purity (lower) categories, with tight (left) and loose (right) b tagging selections. The preferred background-only fit is shown as a solid blue line with an associated shaded band indicating the uncertainty. An alternative fit is shown as a purple dashed line. The ratio panels show the pulls in each bin,

the eight categories. In the fit, which is based on a profile likelihood, the shape parameters and the normalization of the background in each category are free to float. Systematic uncertainties are treated as nuisance parameters and are profiled in the statistical interpretation [64]. The backgroundonly hypothesis is tested against the signal hypothesis in the eight exclusive categories simultaneously. The asymptotic
$\left(N^{\text {data }}-N^{\text {bkg }}\right) / \sigma$, where $\sigma$ is the Poisson uncertainty in data. The horizontal bars on the data points indicate the bin width and the vertical bars represent the normalized Poisson errors, and are shown also for bins with zero entries up to the highest $m_{\mathrm{VH}}$ event. The expected contribution of a resonance with $m_{\mathrm{X}}=2000 \mathrm{GeV}$, simulated in the context of the HVT model B, is shown as a dot-dashed red line

modified frequentist method [65] is used to determine limits at $95 \% \mathrm{CL}$ on the contribution from signal $[66,67]$. Limits are derived on the product of the cross section for a heavy vector boson $\mathrm{X}$ and the branching fractions for the decays $\mathrm{X} \rightarrow \mathrm{VH}$ and $\mathrm{H} \rightarrow \mathrm{b} \overline{\mathrm{b}}$, denoted $\sigma(\mathrm{X}) \mathcal{B}(\mathrm{X} \rightarrow \mathrm{VH}) \mathcal{B}(\mathrm{H} \rightarrow \mathrm{b} \overline{\mathrm{b}})$.

Results are given in the spin-1 hypothesis both for $\mathrm{W}^{\prime} \rightarrow$ $\mathrm{WH}$ and $\mathrm{Z}^{\prime} \rightarrow \mathrm{ZH}$ separately (Fig. 5) as well as for the 

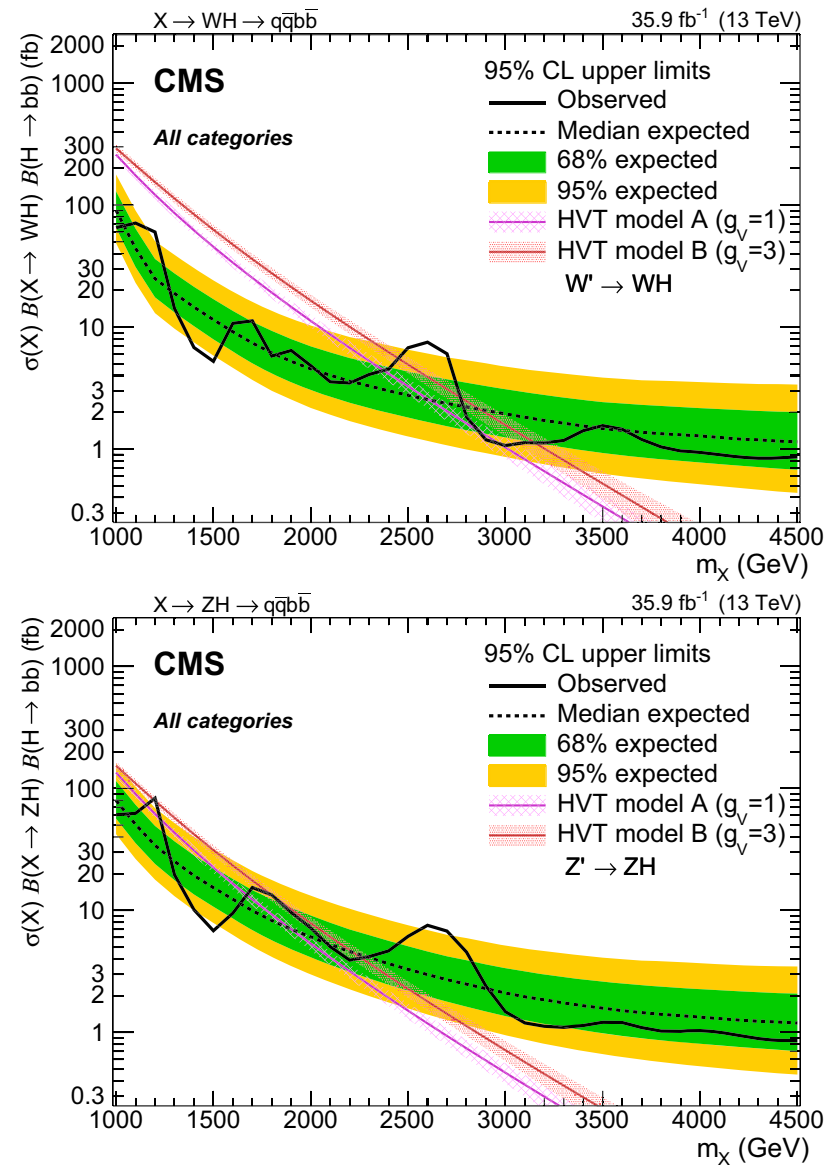

Fig. 5 Observed and expected 95\% CL upper limits on the product $\sigma(\mathrm{X}) \mathcal{B}(\mathrm{X} \rightarrow \mathrm{WH}) \mathcal{B}(\mathrm{H} \rightarrow \mathrm{b} \overline{\mathrm{b}})$ (upper) and $\sigma(\mathrm{X}) \mathcal{B}(\mathrm{X} \rightarrow$ $\mathrm{ZH}) \mathcal{B}(\mathrm{H} \rightarrow \mathrm{b} \bar{b})$ (lower) as a function of the resonance mass for a single narrow spin-1 resonance, for the combination of the eight categories, and including all statistical and systematic uncertainties. The inner green and outer yellow bands represent the \pm 1 and \pm 2 standard deviation uncertainties in the expected limit. The purple and red solid curves correspond to the cross sections predicted by the HVT model A and model B, respectively

heavy vector triplet hypothesis $\mathrm{V}^{\prime} \rightarrow \mathrm{VH}$ summing the massdegenerate $\mathrm{W}^{\prime}$ and $\mathrm{Z}^{\prime}$ production cross sections together (Fig. 6), where they are compared to the cross sections expected in HVT models A and B. Upper limits in the range $0.9-90 \mathrm{fb}$ are set on the product of the cross section and the combined branching fraction for its decay to a vector boson and a Higgs boson decaying into a pair of b quarks, and compared to the HVT models A and B. In this case, the value of $\mathcal{B}(\mathrm{H} \rightarrow \mathrm{b} \bar{b})$ is assumed to be $0.5824 \pm 0.008$ [68]. The uncertainties in the signal normalization from PDFs, and factorization and renormalization scales, are not profiled in the likelihood fit, as they are reported separately as uncertainties in the model cross section. From the combination of the eight categories, a narrow $\mathrm{W}^{\prime}$ resonance with $m_{\mathrm{W}^{\prime}}<2.37 \mathrm{TeV}$ and $2.87<m_{\mathrm{W}^{\prime}}<2.97 \mathrm{TeV}$ can be excluded at $95 \%$ $\mathrm{CL}$ in model $\mathrm{A}$, and $m_{\mathrm{W}^{\prime}}<3.15 \mathrm{TeV}$ except in a region

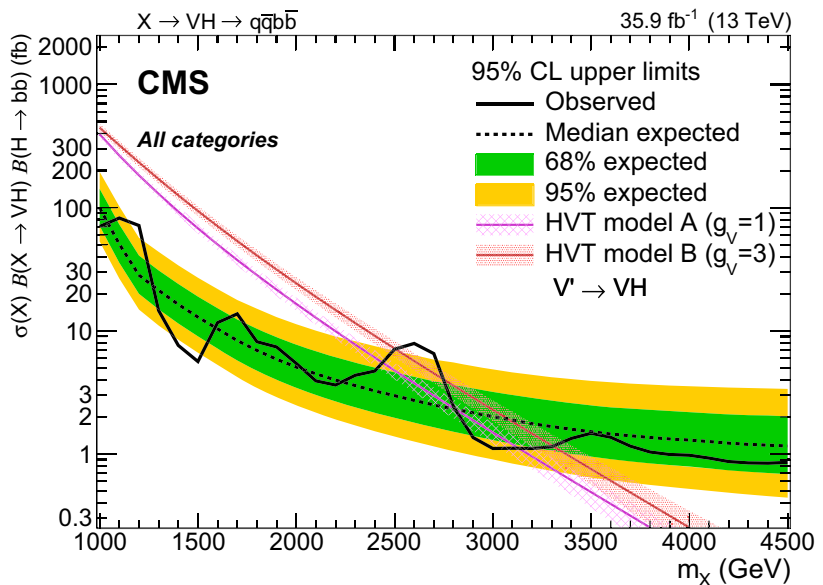

Fig. 6 Observed and expected 95\% CL upper limits with the \pm 1 and \pm 2 standard deviation uncertainty bands on the product $\sigma(\mathrm{X}) \mathcal{B}(\mathrm{X} \rightarrow$ $\mathrm{VH}) \mathcal{B}(\mathrm{H} \rightarrow \mathrm{b} \bar{b})$ in the combined heavy vector triplet hypothesis, for the combination of the eight categories. The purple and red solid curves correspond to the cross sections predicted by the HVT model A and model B, respectively

between 2.45 and $2.78 \mathrm{TeV}$ in model $\mathrm{B}$. A $\mathrm{Z}^{\prime}$ resonance with $m_{\mathrm{Z}^{\prime}}<1.15 \mathrm{TeV}$ or $1.25<m_{\mathrm{Z}^{\prime}}<1.67 \mathrm{TeV}$ is excluded in the HVT model A, and the ranges $m_{\mathrm{Z}^{\prime}}<1.19 \mathrm{TeV}$ and $1.21<m_{\mathrm{Z}^{\prime}}<2.26 \mathrm{TeV}$ are excluded in model $\mathrm{B}$.

The excluded regions for the HVT masses are 1.00$2.43 \mathrm{TeV}$ and $2.81-3.13 \mathrm{TeV}$ in the benchmark model A. The ranges excluded in the framework of model B are 1.00 2.50 and $2.76-3.30 \mathrm{TeV}$, significantly extending the reach with respect to the previous $\sqrt{s}=8 \mathrm{TeV}$ and $\sqrt{s}=13 \mathrm{TeV}$ searches $[20,24]$. The largest observed excess, according to the modified frequentist CLs method [67], corresponds to a mass of $2.6 \mathrm{TeV}$ and has a local (global) significance of 2.6 (0.9) standard deviations.

The exclusion limit shown in Fig. 6 can be interpreted as a function of the coupling strength of the heavy vectors to the $\mathrm{SM}$ bosons and fermions in the $\left[g_{\mathrm{V}} c_{\mathrm{H}}, g^{2} c_{\mathrm{F}} / g_{\mathrm{V}}\right]$ plane. Here, the uncertainties in the signal normalization from PDFs, and factorization and renormalization scales, are profiled in the fit. The excluded region of the parameter space for narrow resonances determined with an analysis of the combined eight categories of data is shown in Fig. 7. The region of the parameter space where the natural width of the resonances exceeds the typical experimental width of $4 \%$, and thus invalidates the narrow width approximation, is also indicated in Fig. 7.

\section{Summary}

A search for a heavy resonance with a mass above $1 \mathrm{TeV}$ and decaying into a vector boson and a Higgs boson, has been presented. The search is based on the final states associated 


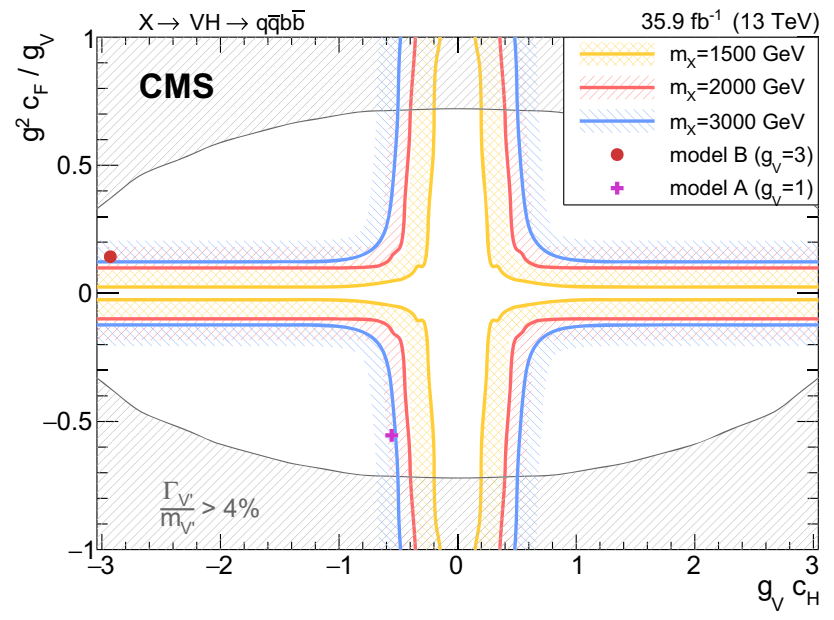

Fig. 7 Observed exclusion in the HVT parameter plane $\left[g_{\mathrm{V}} c_{\mathrm{H}}, g^{2} c_{\mathrm{F}} / g_{\mathrm{V}}\right]$ for three different resonance masses $(1.5,2.0$, and $3.0 \mathrm{TeV}$ ). The parameter $g_{\mathrm{V}}$ represents the coupling strength of the new interaction, $c_{\mathrm{H}}$ the coupling between the HVT bosons and the Higgs boson and longitudinally polarized SM vector bosons, and $c_{\mathrm{F}}$ the coupling between the heavy vector bosons and the SM fermions. The benchmark scenarios corresponding to HVT model A and model B are represented by a purple cross and a red point. The gray shaded areas correspond to the region where the resonance natural width is predicted to be larger than the typical experimental resolution (4\%) and thus the narrow-width approximation does not apply

with the hadronic decay modes of the vector boson and the decay mode of the Higgs boson to $a \mathrm{~b} \bar{b}$ pair. The data sample was collected by the CMS experiment at $\sqrt{s}=13 \mathrm{TeV}$ during 2016, and corresponds to an integrated luminosity of $35.9 \mathrm{fb}^{-1}$. Within the framework of the heavy vector triplet model, mass-dependent upper limits in the range 0.9-90 fb are set on the product of the cross section for production of a narrow spin-1 resonance and the combined branching fraction for its decay to a vector boson and a Higgs boson decaying into a pair of $\mathrm{b}$ quarks. Compared to previous measurements, the range of resonance masses excluded within the framework of benchmark model B of the heavy vector triplet model is extended substantially to values as high as 3.3 TeV. More generally, the results lead to a significant reduction in the allowed parameter space for heavy vector triplet models.

Acknowledgements We congratulate our colleagues in the CERN accelerator departments for the excellent performance of the LHC and thank the technical and administrative staffs at CERN and at other CMS institutes for their contributions to the success of the CMS effort. In addition, we gratefully acknowledge the computing centers and personnel of the Worldwide LHC Computing Grid for delivering so effectively the computing infrastructure essential to our analyses. Finally, we acknowledge the enduring support for the construction and operation of the LHC and the CMS detector provided by the following funding agencies: BMWFW and FWF (Austria); FNRS and FWO (Belgium); CNPq, CAPES, FAPERJ, and FAPESP (Brazil); MES (Bulgaria); CERN; CAS, MoST, and NSFC (China); COLCIENCIAS (Colombia); MSES and CSF (Croatia); RPF (Cyprus); SENESCYT
(Ecuador); MoER, ERC IUT, and ERDF (Estonia); Academy of Finland, MEC, and HIP (Finland); CEA and CNRS/IN2P3 (France); BMBF, DFG, and HGF (Germany); GSRT (Greece); OTKA and NIH (Hungary); DAE and DST (India); IPM (Iran); SFI (Ireland); INFN (Italy); MSIP and NRF (Republic of Korea); LAS (Lithuania); MOE and UM (Malaysia); BUAP, CINVESTAV, CONACYT, LNS, SEP, and UASLP-FAI (Mexico); MBIE (New Zealand); PAEC (Pakistan); MSHE and NSC (Poland); FCT (Portugal); JINR (Dubna); MON, RosAtom, RAS, RFBR and RAEP (Russia); MESTD (Serbia); SEIDI, CPAN, PCTI and FEDER (Spain); Swiss Funding Agencies (Switzerland); MST (Taipei); ThEPCenter, IPST, STAR, and NSTDA (Thailand); TUBITAK and TAEK (Turkey); NASU and SFFR (Ukraine); STFC (UK); DOE and NSF (USA). Individuals have received support from the Marie-Curie program and the European Research Council and Horizon 2020 Grant, contract No. 675440 (European Union); the Leventis Foundation; the A. P. Sloan Foundation; the Alexander von Humboldt Foundation; the Belgian Federal Science Policy Office; the Fonds pour la Formation à la Recherche dans l'Industrie et dans l'Agriculture (FRIA-Belgium); the Agentschap voor Innovatie door Wetenschap en Technologie (IWT-Belgium); the Ministry of Education, Youth and Sports (MEYS) of the Czech Republic; the Council of Science and Industrial Research, India; the HOMING PLUS program of the Foundation for Polish Science, cofinanced from European Union, Regional Development Fund, the Mobility Plus program of the Ministry of Science and Higher Education, the National Science Center (Poland), contracts Harmonia 2014/14/M/ST2/00428, Opus 2014/13/B/ST2/02543, 2014/15/B/ST2/03998, and 2015/19/B/ST2/02861, Sonata-bis 2012/07/ E/ST2/01406; the National Priorities Research Program by Qatar National Research Fund; the Programa Clarín-COFUND del Principado de Asturias; the Thalis and Aristeia programs cofinanced by EUESF and the Greek NSRF; the Rachadapisek Sompot Fund for Postdoctoral Fellowship, Chulalongkorn University and the Chulalongkorn Academic into its 2nd Century Project Advancement Project (Thailand); and the Welch Foundation, contract C-1845.

Open Access This article is distributed under the terms of the Creative Commons Attribution 4.0 International License (http://creativecomm ons.org/licenses/by/4.0/), which permits unrestricted use, distribution, and reproduction in any medium, provided you give appropriate credit to the original author(s) and the source, provide a link to the Creative Commons license, and indicate if changes were made. Funded by SCOAP . $^{3}$

\section{References}

1. ATLAS Collaboration, Observation of a new particle in the search for the Standard Model Higgs boson with the ATLAS detector at the LHC. Phys. Lett. B 716, 1 (2012). doi:10.1016/j.physletb.2012. 08.020. arXiv: 1207.7214

2. CMS Collaboration, Observation of a new boson at a mass of 125 $\mathrm{GeV}$ with the CMS experiment at the LHC. Phys. Lett. B 716, 30 (2012). doi:10.1016/j.physletb.2012.08.021. arXiv:1207.7235

3. CMS Collaboration, Observation of a new boson with mass near $125 \mathrm{GeV}$ in pp collisions at $\sqrt{s}=7$ and $8 \mathrm{TeV}$. JHEP 06, 081 (2013). doi:10.1007/JHEP06(2013)081. arXiv:1303.4571

4. ATLAS Collaboration, Measurement of the Higgs boson mass from the $H \rightarrow \gamma \gamma$ and $H \rightarrow Z Z^{*} \rightarrow 4 \ell$ channels in $p p$ collisions at center-of-mass energies of 7 and $8 \mathrm{TeV}$ with the ATLAS detector. Phys. Rev. D 90, 052004 (2014). doi:10.1103/PhysRevD.90. 052004. arXiv: 1406.3827

5. CMS Collaboration, Precise determination of the mass of the Higgs boson and tests of compatibility of its couplings with the standard model predictions using proton collisions at 7 and $8 \mathrm{TeV}$. Eur. 
Phys. J. C 75, 212 (2015). doi:10.1140/epjc/s10052-015-3351-7. arXiv: 1412.8662

6. CMS Collaboration, Evidence for the direct decay of the $125 \mathrm{GeV}$ Higgs boson to fermions. Nat. Phys. 10, 557 (2014). doi:10.1038/ nphys3005. arXiv: 1401.6527

7. ATLAS and CMS Collaborations, Combined measurement of the Higgs boson mass in $p p$ collisions at $\sqrt{s}=7$ and $8 \mathrm{TeV}$ with the ATLAS and CMS experiments. Phys. Rev. Lett. 114, 191803 (2015). doi:10.1103/PhysRevLett.114.191803. arXiv:1503.07589

8. V.D. Barger, W.-Y. Keung, E. Ma, A gauge model with light $W$ and $Z$ bosons. Phys. Rev. D 22, 727 (1980). doi:10.1103/PhysRevD. 22.727

9. E. Salvioni, G. Villadoro, F. Zwirner, Minimal Z' models: present bounds and early LHC reach. JHEP 09, 068 (2009). doi:10.1088/ 1126-6708/2009/11/068. arXiv:0909.1320

10. C. Grojean, E. Salvioni, R. Torre, A weakly constrained $\mathrm{W}^{\prime}$ at the early LHC. JHEP 07, 002 (2011). doi:10.1007/JHEP07(2011)002. arXiv: 1103.2761

11. R. Contino, D. Pappadopulo, D. Marzocca, R. Rattazzi, On the effect of resonances in composite higgs phenomenology. JHEP 10, 081 (2011). doi:10.1007/JHEP10(2011)081. arXiv:1109.1570

12. D. Marzocca, M. Serone, J. Shu, General composite Higgs models. JHEP 08, 13 (2012). doi:10.1007/JHEP08(2012)013. arXiv: 1205.0770

13. B. Bellazzini, C. Csaki, J. Serra, Composite Higgses. Eur. Phys. J. C 74, 2766 (2014). doi:10.1140/epjc/s10052-014-2766-x. arXiv: 1401.2457

14. T. Han, H.E. Logan, B. McElrath, L.-T. Wang, Phenomenology of the little Higgs model. Phys. Rev. D 67, 095004 (2003). doi:10. 1103/PhysRevD.67.095004. arXiv:hep-ph/0301040

15. M. Schmaltz, D. Tucker-Smith, Little Higgs theories. Ann. Rev. Nucl. Part. Sci. 55, 229 (2005). doi:10.1146/annurev.nucl.55. 090704.151502. arXiv:hep-ph/0502182

16. M. Perelstein, Little Higgs models and their phenomenology. Prog. Part. Nucl. Phys. 58, 247 (2007). doi:10.1016/j.ppnp.2006.04.001. arXiv:hep-ph/0512128

17. D. Pappadopulo, A. Thamm, R. Torre, A. Wulzer, Heavy vector triplets: bridging theory and data. JHEP 09, 60 (2014). doi:10. 1007/JHEP09(2014)060. arXiv:1402.4431

18. CMS Collaboration, Search for a pseudoscalar boson decaying into a $\mathrm{Z}$ boson and the $125 \mathrm{GeV}$ Higgs boson in $\ell^{+} \ell^{-} b \bar{b}$ final states. Phys. Lett. B 748, 221 (2015). doi:10.1016/j.physletb.2015.07.010. arXiv: 1504.04710

19. ATLAS Collaboration, Search for a new resonance decaying to a $\mathrm{W}$ or Z boson and a Higgs boson in the $\ell \ell / \ell v / \nu v+b \bar{b}$ final states with the ATLAS detector. Eur. Phys. J. C 75, 263 (2015). doi:10. 1140/epjc/s10052-015-3474-x. arXiv:1503.08089

20. CMS Collaboration, Search for a massive resonance decaying into a Higgs boson and a $\mathrm{W}$ or $\mathrm{Z}$ boson in hadronic final states in protonproton collisions at $\sqrt{s}=8 \mathrm{TeV}$. JHEP 02, 145 (2016). doi:10. 1007/JHEP02(2016)145. arXiv:1506.01443

21. CMS Collaboration, Search for narrow high-mass resonances in proton-proton collisions at $\sqrt{s}=8 \mathrm{TeV}$ decaying to a $\mathrm{Z}$ and a Higgs boson. Phys. Lett. B 748, 255 (2015). doi:10.1016/j.physletb.2015. 07.011. arXiv: 1502.04994

22. CMS Collaboration, "Search for massive resonances decaying into $\mathrm{WW}, \mathrm{WZ}$ or ZZ bosons in proton-proton collisions at $\sqrt{s}=$ 13TeV”. JHEP 03, 162 (2017). doi:10.1007/JHEP03(2017)162. arXiv: 1612.09159

23. ATLAS Collaboration, Searches for heavy diboson resonances in $p p$ collisions at $\sqrt{s}=13 \mathrm{TeV}$ with the ATLAS detector. JHEP 09, 173 (2016). doi:10.1007/JHEP09(2016)173. arXiv:1606.04833

24. CMS Collaboration, Search for heavy resonances decaying into a vector boson and a Higgs boson in final states with charged leptons, neutrinos, and b quarks. Phys. Lett. B 768, 137 (2017). doi:10.1016/ j.physletb.2017.02.040. arXiv:1610.08066
25. ATLAS Collaboration, Search for new resonances decaying to a $W$ or $Z$ boson and a Higgs boson in the $\ell^{+} \ell^{-} b \bar{b}, \ell v b \bar{b}$, and $\nu \bar{v} b \bar{b}$ channels with $p p$ collisions at $\sqrt{s}=13 \mathrm{TeV}$ with the ATLAS detector. Phys. Lett. B 765, 32 (2016). doi:10.1016/j.physletb.2016. 11.045. arXiv: 1607.05621

26. J. Alwall et al., The automated computation of tree-level and nextto-leading order differential cross sections, and their matching to parton shower simulations. JHEP 07, 079 (2014). doi:10.1007/ JHEP07(2014)079. arXiv:1405.0301

27. P. Nason, A new method for combining NLO QCD with shower Monte Carlo algorithms. JHEP 11, 040 (2004). doi:10.1088/ 1126-6708/2004/11/040. arXiv:hep-ph/0409146

28. S. Frixione, P. Nason, C. Oleari, Matching NLO QCD computations with Parton Shower simulations: the POWHEG method. JHEP 11, 070 (2007). doi:10.1088/1126-6708/2007/11/070. arXiv:0709.2092

29. S. Alioli, P. Nason, C. Oleari, E. Re, A general framework for implementing NLO calculations in shower Monte Carlo programs: the POWHEG BOX. JHEP 06, 043 (2010). doi:10.1007/ JHEP06(2010)043. arXiv:1002.2581

30. M. Czakon, A. Mitov, Top++: a program for the calculation of the top-pair cross-section at hadron colliders. Comput. Phys. Commun. 185, 2930 (2014). doi:10.1016/j.cpc.2014.06.021. arXiv: 1112.5675

31. R. Frederix, S. Frixione, Merging meets matching in MC@ NLO. JHEP 12, 061 (2012). doi:10.1007/JHEP12(2012)061. arXiv: 1209.6215

32. T. Sjöstrand, S. Mrenna, P. Skands, A brief introduction to PYTHIA 8.1. Comput. Phys. Commun. 178, 852 (2008). doi:10.1016/j.cpc. 2008.01.036. arXiv:0710.3820

33. P. Skands, S. Carrazza, J. Rojo, Tuning PYTHIA 8.1: the Monash, Tune. Eur. Phys. J. C 74(2014), 3024 (2013). doi:10.1140/epjc/ s10052-014-3024-y. arXiv:1404.5630

34. CMS Collaboration, Event generator tunes obtained from underlying event and multiparton scattering measurements. Eur. Phys. J. C 76, 155 (2016). doi:10.1140/epjc/s10052-016-3988-x. arXiv: 1512.00815

35. CMS Collaboration, Investigations of the impact of the parton shower tuning in Pythia 8 in the modelling of $t \bar{t}$ at $\sqrt{s}=8$ and 13 TeV. CMS Physics Analysis Summary CMS-PAS-TOP-16-021. https://cds.cern.ch/record/2235192

36. NNPDF Collaboration, Parton distributions for the LHC Run II. JHEP 04, 040 (2015). doi:10.1007/JHEP04(2015)040. arXiv: 1410.8849

37. GEANT4 Collaboration, GEANT4-a simulation toolkit. Nucl. Instrum. Methods A 506, 250 (2003). doi:10.1016/ S0168-9002(03)01368-8

38. CMS Collaboration, Description and performance of track and primary-vertex reconstruction with the CMS tracker. JINST 9, P10009 (2014), doi:10.1088/1748-0221/9/10/P10009. arXiv: 1405.6569

39. CMS Collaboration, The CMS trigger system. JINST 12, P01020 (2017). doi:10.1088/1748-0221/12/01/P01020. arXiv:1609.02366

40. CMS Collaboration, The CMS experiment at the CERN LHC. JINST 3, S08004 (2008). doi:10.1088/1748-0221/3/08/S08004

41. CMS Collaboration, Particle-flow event reconstruction in CMS and performance for jets, taus, and $E_{\mathrm{T}}^{\text {miss }}$. CMS Physics Analysis Summary CMS-PAS-PFT-09-001, CERN (2009). http://cdsweb.cern. ch/record/1194487

42. CMS Collaboration, Commissioning of the particle-flow event with the first LHC collisions recorded in the CMS detector. CMS Physics Analysis Summary CMS-PAS-PFT-10-001, CERN (2010). http:// cdsweb.cern.ch/record/1247373

43. M. Cacciari, G.P. Salam, G. Soyez, The anti- $k_{\mathrm{t}}$ jet clustering algorithm. JHEP 04, 063 (2008). doi:10.1088/1126-6708/2008/04/063. arXiv:0802.1189 
44. M. Cacciari, G.P. Salam, G. Soyez, FastJet user manual. Eur. Phys. J. C 72, 1896 (2012). doi:10.1140/epjc/s10052-012-1896-2. arXiv: 1111.6097

45. CMS Collaboration, Pileup removal algorithms. CMS Physics Analysis Summary CMS-PAS-JME-14-001, CERN (2014). http:// cds.cern.ch/record/1751454

46. M. Cacciari, G.P. Salam, G. Soyez, The catchment area of jets. JHEP 04, 005 (2008). doi:10.1088/1126-6708/2008/04/005. arXiv:0802.1188

47. CMS Collaboration, Determination of jet energy calibration and transverse momentum resolution in CMS. JINST 6, P11002 (2011). doi:10.1088/1748-0221/6/11/P11002. arXiv:1107.4277

48. CMS Collaboration, Jet energy scale and resolution in the CMS experiment in pp collisions at $8 \mathrm{TeV}$. JINST 12, P02014 (2017). doi:10.1088/1748-0221/12/02/P02014. arXiv:1607.03663

49. CMS Collaboration, Performance of missing energy reconstruction in $13 \mathrm{TeV}$ pp collision data using the CMS detector. CMS Physics Analysis Summary CMS-PAS-JME-16-004, CERN (2016). http:// cds.cern.ch/record/1479660

50. D. Bertolini, P. Harris, M. Low, N. Tran, Pileup per particle identification. JHEP 10, 59 (2014). doi:10.1007/JHEP10(2014)059. arXiv:1407.6013

51. M. Dasgupta, A. Fregoso, S. Marzani, G.P. Salam, Towards an understanding of jet substructure. JHEP 09, 029 (2013). doi:10. 1007/JHEP09(2013)029. arXiv:1307.0007

52. A.J. Larkoski, S. Marzani, G. Soyez, J. Thaler, Soft drop. JHEP 05, 146 (2014). doi:10.1007/JHEP05(2014)146. arXiv:1402.2657

53. CMS Collaboration, Jet algorithms performance in $13 \mathrm{TeV}$ data. CMS Physics Analysis Summary CMS-PAS-JME-16-003, CERN (2017). http://cds.cern.ch/record/2256875

54. J. Thaler, K. Van Tilburg, Identifying boosted objects with N-subjettiness. JHEP 03, 015 (2011). doi:10.1007/ JHEP03(2011)015. arXiv:1011.2268

55. CMS Collaboration, Identification of double-b quark jets in boosted event topologies. CMS Physics Analysis Summary CMS-PASBTV-15-002, CERN (2016)

56. CMS Collaboration, Performance of electron reconstruction and selection with the CMS detector in proton-proton collisions at $\sqrt{s}=8$ TeV. JINST 10, P06005 (2015). doi:10.1088/1748-0221/ 10/06/P06005. arXiv:1502.02701

57. CMS Collaboration, Performance of CMS muon reconstruction in pp collision events at $\sqrt{s}=7 \mathrm{TeV}$. JINST 7, P10002 (2012). doi:10. 1088/1748-0221/7/10/P10002. arXiv:1206.4071

58. CMS Collaboration, Reconstruction and identification of $\tau$ lepton decays to hadrons and $v_{\tau}$ at CMS. JINST 11, P01019 (2016). doi:10.1088/1748-0221/11/01/P01019. arXiv:1510.07488

59. D. Krohn, J. Thaler, L.-T. Wang, Jet trimming. JHEP 02, 084 (2010). doi:10.1007/JHEP02(2010)084. arXiv:0912.1342

60. R.A. Fisher, Statistical methods for research workers (Oliver and Boyd, 1954) (ISBN: 0-05-002170-2)

61. M. Bähr et al., Herwig++ physics and manual. Eur. Phys. J. C 58, 639 (2008). doi:10.1140/epjc/s10052-008-0798-9. arXiv:0803.0883

62. CMS Collaboration, CMS luminosity measurement for the 2016 data taking period. CMS Physics Analysis Summary CMS-PASLUM-17-001, CERN (2017). http://cds.cern.ch/record/2257069

63. J. Butterworth et al., PDF4LHC recommendations for LHC Run II. J. Phys. G 43, 23001 (2016). doi:10.1088/0954-3899/43/2/023001. arXiv: 1510.03865

64. CMS and ATLAS Collaborations, Procedure for the LHC Higgs boson search combination in Summer 2011. CMS Note CMS-NOTE-2011-005, ATL-PHYS-PUB-2011-11, CERN (2011). https://cds.cern.ch/record/1379837

65. G. Cowan, K. Cranmer, E. Gross, O. Vitells, Asymptotic formulae for likelihood-based tests of new physics. Eur. Phys. J. C 71, 1554 (2011). doi:10.1140/epjc/s10052-011-1554-0. arXiv:1007.1727 (Erratum: doi: 10.1140/epjc/s10052-013-2501-z)

66. T. Junk, Confidence level computation for combining searches with small statistics. Nucl. Instrum. Methods A 434, 435 (1999). doi:10. 1016/S0168-9002(99)00498-2. arXiv:hep-ex/9902006

67. A.L. Read, Presentation of search results: the $C L_{s}$ technique. J. Phys. G 28, 2693 (2002). doi:10.1088/0954-3899/28/10/313

68. D. de Florian et al., Handbook of LHC Higgs cross sections: 4. deciphering the nature of the Higgs sector. CERN Yellow Report CERN-2017-002-M, CERN (2016). doi:10.23731/ CYRM-2017-002. arXiv:1610.07922

\section{CMS Collaboration}

\section{Yerevan Physics Institute, Yerevan, Armenia}

A. M. Sirunyan, A. Tumasyan

\section{Institut für Hochenergiephysik, Vienna, Austria}

W. Adam, F. Ambrogi, E. Asilar, T. Bergauer, J. Brandstetter, E. Brondolin, M. Dragicevic, J. Erö, M. Flechl, M. Friedl, R. Frühwirth ${ }^{1}$, V. M. Ghete, J. Grossmann, J. Hrubec, M. Jeitler ${ }^{1}$, A. König, N. Krammer, I. Krätschmer, D. Liko,

T. Madlener, I. Mikulec, E. Pree, D. Rabady, N. Rad, H. Rohringer, J. Schieck ${ }^{1}$, R. Schöfbeck, M. Spanring, D. Spitzbart, J. Strauss, W. Waltenberger, J. Wittmann, C.-E. Wulz ${ }^{1}$, M. Zarucki

\section{Institute for Nuclear Problems, Minsk, Belarus}

V. Chekhovsky, V. Mossolov, J. Suarez Gonzalez

\section{Universiteit Antwerpen, Antwerpen, Belgium}

E. A. De Wolf, D. Di Croce, X. Janssen, J. Lauwers, H. Van Haevermaet, P. Van Mechelen, N. Van Remortel

\section{Vrije Universiteit Brussel, Brussel, Belgium}

S. Abu Zeid, F. Blekman, J. D'Hondt, I. De Bruyn, J. De Clercq, K. Deroover, G. Flouris, D. Lontkovskyi, S. Lowette, S. Moortgat, L. Moreels, A. Olbrechts, Q. Python, K. Skovpen, S. Tavernier, W. Van Doninck, P. Van Mulders, I. Van Parijs

\section{Université Libre de Bruxelles, Brussels, Belgium}

H. Brun, B. Clerbaux, G. De Lentdecker, H. Delannoy, G. Fasanella, L. Favart, R. Goldouzian, A. Grebenyuk, 
G. Karapostoli, T. Lenzi, J. Luetic, T. Maerschalk, A. Marinov, A. Randle-conde, T. Seva, C. Vander Velde, P. Vanlaer,

D. Vannerom, R. Yonamine, F. Zenoni, F. Zhang ${ }^{2}$

\section{Ghent University, Ghent, Belgium}

A. Cimmino, T. Cornelis, D. Dobur, A. Fagot, M. Gul, I. Khvastunov, D. Poyraz, C. Roskas, S. Salva, M. Tytgat,

W. Verbeke, N. Zaganidis

Université Catholique de Louvain, Louvain-la-Neuve, Belgium

H. Bakhshiansohi, O. Bondu, S. Brochet, G. Bruno, A. Caudron, S. De Visscher, C. Delaere, M. Delcourt, B. Francois,

A. Giammanco, A. Jafari, M. Komm, G. Krintiras, V. Lemaitre, A. Magitteri, A. Mertens, M. Musich, K. Piotrzkowski,

L. Quertenmont, M. Vidal Marono, S. Wertz

Université de Mons, Mons, Belgium

N. Beliy

Centro Brasileiro de Pesquisas Fisicas, Rio de Janeiro, Brazil

W. L. Aldá Júnior, F. L. Alves, G. A. Alves, L. Brito, M. Correa Martins Junior, C. Hensel, A. Moraes, M. E. Pol,

P. Rebello Teles

Universidade do Estado do Rio de Janeiro, Rio de Janeiro, Brazil

E. Belchior Batista Das Chagas, W. Carvalho, J. Chinellato ${ }^{3}$, A. Custódio, E. M. Da Costa, G. G. Da Silveira ${ }^{4}$,

D. De Jesus Damiao, S. Fonseca De Souza, L. M. Huertas Guativa, H. Malbouisson, M. Melo De Almeida, C. Mora Herrera,

L. Mundim, H. Nogima, A. Santoro, A. Sznajder, E. J. Tonelli Manganote ${ }^{3}$, F. Torres Da Silva De Araujo, A. Vilela Pereira

Universidade Estadual Paulista ${ }^{a}$, Universidade Federal do $\mathrm{ABC}^{b}$, São Paulo, Brazil

S. Ahuja ${ }^{a}$, C. A. Bernardes ${ }^{a}$, T. R. Fernandez Perez Tomei ${ }^{a}$, E. M. Gregores ${ }^{b}$, P. G. Mercadante ${ }^{b}$, S. F. Novaes ${ }^{a}$,

Sandra S. Padula ${ }^{a}$, D. Romero $\operatorname{Abad}^{b}$, J. C. Ruiz Vargas ${ }^{a}$

Institute for Nuclear Research and Nuclear Energy of Bulgaria Academy of Sciences, Sofia, Bulgaria

A. Aleksandrov, R. Hadjiiska, P. Iaydjiev, M. Misheva, M. Rodozov, M. Shopova, S. Stoykova, G. Sultanov

University of Sofia, Sofia, Bulgaria

A. Dimitrov, I. Glushkov, L. Litov, B. Pavlov, P. Petkov

Beihang University, Beijing, China

W. Fang ${ }^{5}$, X. Gao ${ }^{5}$

Institute of High Energy Physics, Beijing, China

M. Ahmad, J. G. Bian, G. M. Chen, H. S. Chen, M. Chen, Y. Chen, C. H. Jiang, D. Leggat, H. Liao, Z. Liu, F. Romeo, S.

M. Shaheen, A. Spiezia, J. Tao, C. Wang, Z. Wang, E. Yazgan, H. Zhang, J. Zhao

State Key Laboratory of Nuclear Physics and Technology, Peking University, Beijing, China

Y. Ban, G. Chen, Q. Li, S. Liu, Y. Mao, S. J. Qian, D. Wang, Z. Xu

Universidad de Los Andes, Bogota, Colombia

C. Avila, A. Cabrera, L. F. Chaparro Sierra, C. Florez, C. F. González Hernández, J. D. Ruiz Alvarez

Faculty of Electrical Engineering, Mechanical Engineering and Naval Architecture, University of Split, Split, Croatia

B. Courbon, N. Godinovic, D. Lelas, I. Puljak, P. M. Ribeiro Cipriano, T. Sculac

Faculty of Science, University of Split, Split, Croatia

Z. Antunovic, M. Kovac

Institute Rudjer Boskovic, Zagreb, Croatia

V. Brigljevic, D. Ferencek, K. Kadija, B. Mesic, A. Starodumov ${ }^{6}$, T. Susa

University of Cyprus, Nicosia, Cyprus

M. W. Ather, A. Attikis, G. Mavromanolakis, J. Mousa, C. Nicolaou, F. Ptochos, P. A. Razis, H. Rykaczewski

Charles University, Prague, Czech Republic

M. Finger ${ }^{7}$, M. Finger Jr. ${ }^{7}$ 
Universidad San Francisco de Quito, Quito, Ecuador

E. Carrera Jarrin

Egyptian Network of High Energy Physics, Academy of Scientific Research and Technology of the Arab Republic of Egypt, Cairo, Egypt

A. A. Abdelalim ${ }^{8,9}$, Y. Mohammed ${ }^{10}$, E. Salama ${ }^{11,12}$

National Institute of Chemical Physics and Biophysics, Tallinn, Estonia

R. K. Dewanjee, M. Kadastik, L. Perrini, M. Raidal, A. Tiko, C. Veelken

Department of Physics, University of Helsinki, Helsinki, Finland

P. Eerola, J. Pekkanen, M. Voutilainen

Helsinki Institute of Physics, Helsinki, Finland

J. Härkönen, T. Järvinen, V. Karimäki, R. Kinnunen, T. Lampén, K. Lassila-Perini, S. Lehti, T. Lindén, P. Luukka,

E. Tuominen, J. Tuominiemi, E. Tuovinen

Lappeenranta University of Technology, Lappeenranta, Finland

J. Talvitie, T. Tuuva

IRFU, CEA, Université Paris-Saclay, Gif-sur-Yvette, France

M. Besancon, F. Couderc, M. Dejardin, D. Denegri, J. L. Faure, F. Ferri, S. Ganjour, S. Ghosh, A. Givernaud, P. Gras,

G. Hamel de Monchenault, P. Jarry, I. Kucher, E. Locci, M. Machet, J. Malcles, G. Negro, J. Rander, A. Rosowsky, M.

Ö. Sahin, M. Titov

Laboratoire Leprince-Ringuet, Ecole polytechnique, CNRS/IN2P3, Université Paris-Saclay, Palaiseau, France A. Abdulsalam, I. Antropov, S. Baffioni, F. Beaudette, P. Busson, L. Cadamuro, C. Charlot, R. Granier de Cassagnac, M. Jo, S. Lisniak, A. Lobanov, J. Martin Blanco, M. Nguyen, C. Ochando, G. Ortona, P. Paganini, P. Pigard, S. Regnard, R. Salerno, J. B. Sauvan, Y. Sirois, A. G. Stahl Leiton, T. Strebler, Y. Yilmaz, A. Zabi, A. Zghiche

Université de Strasbourg, CNRS, IPHC UMR 7178, 67000 Strasbourg, France

J.-L. Agram ${ }^{13}$, J. Andrea, D. Bloch, J.-M. Brom, M. Buttignol, E. C. Chabert, N. Chanon, C. Collard, E. Conte ${ }^{13}$,

X. Coubez, J.-C. Fontaine ${ }^{13}$, D. Gelé, U. Goerlach, M. Jansová, A.-C. Le Bihan, N. Tonon, P. Van Hove

Centre de Calcul de l'Institut National de Physique Nucleaire et de Physique des Particules, CNRS/IN2P3, Villeurbanne, France

S. Gadrat

Institut de Physique Nucléaire de Lyon, Université de Lyon, Université Claude Bernard Lyon 1, CNRS-IN2P3, Villeurbanne, France

S. Beauceron, C. Bernet, G. Boudoul, R. Chierici, D. Contardo, P. Depasse, H. El Mamouni, J. Fay, L. Finco, S. Gascon, M. Gouzevitch, G. Grenier, B. Ille, F. Lagarde, I. B. Laktineh, M. Lethuillier, L. Mirabito, A. L. Pequegnot, S. Perries, A. Popov ${ }^{14}$, V. Sordini, M. Vander Donckt, S. Viret

Georgian Technical University, Tbilisi, Georgia

T. Toriashvili ${ }^{15}$

Tbilisi State University, Tbilisi, Georgia

Z. Tsamalaidze ${ }^{7}$

RWTH Aachen University, I. Physikalisches Institut, Aachen, Germany

C. Autermann, S. Beranek, L. Feld, M. K. Kiesel, K. Klein, M. Lipinski, M. Preuten, C. Schomakers, J. Schulz, T. Verlage

RWTH Aachen University, III. Physikalisches Institut A, Aachen, Germany

A. Albert, E. Dietz-Laursonn, D. Duchardt, M. Endres, M. Erdmann, S. Erdweg, T. Esch, R. Fischer, A. Güth, M. Hamer, T. Hebbeker, C. Heidemann, K. Hoepfner, S. Knutzen, M. Merschmeyer, A. Meyer, P. Millet, S. Mukherjee,

M. Olschewski, K. Padeken, T. Pook, M. Radziej, H. Reithler, M. Rieger, F. Scheuch, D. Teyssier, S. Thüer 
RWTH Aachen University, III. Physikalisches Institut B, Aachen, Germany

G. Flügge, B. Kargoll, T. Kress, A. Künsken, J. Lingemann, T. Müller, A. Nehrkorn, A. Nowack, C. Pistone, O. Pooth, A. Stahl ${ }^{16}$

\section{Deutsches Elektronen-Synchrotron, Hamburg, Germany}

M. Aldaya Martin, T. Arndt, C. Asawatangtrakuldee, K. Beernaert, O. Behnke, U. Behrens, A. Bermúdez Martínez, A. A. Bin Anuar, K. Borras ${ }^{17}$, V. Botta, A. Campbell, P. Connor, C. Contreras-Campana, F. Costanza, C. Diez Pardos, G. Eckerlin, D. Eckstein, T. Eichhorn, E. Eren, E. Gallo ${ }^{18}$, J. Garay Garcia, A. Geiser, A. Gizhko, J. M. Grados Luyando, A. Grohsjean, P. Gunnellini, A. Harb, J. Hauk, M. Hempel ${ }^{19}$, H. Jung, A. Kalogeropoulos, M. Kasemann, J. Keaveney, C. Kleinwort, I. Korol, D. Krücker, W. Lange, A. Lelek, T. Lenz, J. Leonard, K. Lipka, W. Lohmann ${ }^{19}$, R. Mankel, I.-A. Melzer-Pellmann, A. B. Meyer, G. Mittag, J. Mnich, A. Mussgiller, E. Ntomari, D. Pitzl, R. Placakyte, A. Raspereza, B. Roland, M. Savitskyi, P. Saxena, R. Shevchenko, S. Spannagel, N. Stefaniuk, G. P. Van Onsem, R. Walsh, Y. Wen, K. Wichmann, C. Wissing, O. Zenaiev

University of Hamburg, Hamburg, Germany

S. Bein, V. Blobel, M. Centis Vignali, A. R. Draeger, T. Dreyer, E. Garutti, D. Gonzalez, J. Haller, A. Hinzmann, M. Hoffmann, A. Karavdina, R. Klanner, R. Kogler, N. Kovalchuk, S. Kurz, T. Lapsien, I. Marchesini, D. Marconi, M. Meyer, M. Niedziela, D. Nowatschin, F. Pantaleo ${ }^{16}$, T. Peiffer, A. Perieanu, C. Scharf, P. Schleper, A. Schmidt, S. Schumann, J. Schwandt, J. Sonneveld, H. Stadie, G. Steinbrück, F. M. Stober, M. Stöver, H. Tholen, D. Troendle, E. Usai, L. Vanelderen, A. Vanhoefer, B. Vormwald

Institut für Experimentelle Kernphysik, Karlsruhe, Germany

M. Akbiyik, C. Barth, S. Baur, E. Butz, R. Caspart, T. Chwalek, F. Colombo, W. De Boer, A. Dierlamm, B. Freund, R. Friese, M. Giffels, A. Gilbert, D. Haitz, F. Hartmann ${ }^{16}$, S. M. Heindl, U. Husemann, F. Kassel ${ }^{16}$, S. Kudella, H. Mildner, M. U. Mozer, Th. Müller, M. Plagge, G. Quast, K. Rabbertz, M. Schröder, I. Shvetsov, G. Sieber, H. J. Simonis, R. Ulrich, S. Wayand, M. Weber, T. Weiler, S. Williamson, C. Wöhrmann, R. Wolf

Institute of Nuclear and Particle Physics (INPP), NCSR Demokritos, Aghia Paraskevi, Greece G. Anagnostou, G. Daskalakis, T. Geralis, V. A. Giakoumopoulou, A. Kyriakis, D. Loukas, I. Topsis-Giotis

National and Kapodistrian University of Athens, Athens, Greece

S. Kesisoglou, A. Panagiotou, N. Saoulidou

University of Ioánnina, Ioannina, Greece

I. Evangelou, C. Foudas, P. Kokkas, S. Mallios, N. Manthos, I. Papadopoulos, E. Paradas, J. Strologas, F. A. Triantis

MTA-ELTE Lendület CMS Particle and Nuclear Physics Group, Eötvös Loránd University, Budapest, Hungary M. Csanad, N. Filipovic, G. Pasztor

Wigner Research Centre for Physics, Budapest, Hungary

G. Bencze, C. Hajdu, D. Horvath ${ }^{20}$, Á. Hunyadi, F. Sikler, V. Veszpremi, G. Vesztergombi ${ }^{21}$, A. J. Zsigmond

Institute of Nuclear Research ATOMKI, Debrecen, Hungary

N. Beni, S. Czellar, J. Karancsi ${ }^{22}$, A. Makovec, J. Molnar, Z. Szillasi

Institute of Physics, University of Debrecen, Debrecen, Hungary

M. Bartók ${ }^{21}$, P. Raics, Z. L. Trocsanyi, B. Ujvari

Indian Institute of Science (IISc), Bangalore, India

S. Choudhury, J. R. Komaragiri

National Institute of Science Education and Research, Bhubaneswar, India

S. Bahinipati ${ }^{23}$, S. Bhowmik, P. Mal, K. Mandal, A. Nayak ${ }^{24}$, D. K. Sahoo ${ }^{23}$, N. Sahoo, S. K. Swain

Panjab University, Chandigarh, India

S. Bansal, S. B. Beri, V. Bhatnagar, U. Bhawandeep, R. Chawla, N. Dhingra, A. K. Kalsi, A. Kaur, M. Kaur, R. Kumar, P. Kumari, A. Mehta, J. B. Singh, G. Walia 


\section{University of Delhi, Delhi, India}

Ashok Kumar, Aashaq Shah, A. Bhardwaj, S. Chauhan, B. C. Choudhary, R. B. Garg, S. Keshri, A. Kumar, S. Malhotra, M. Naimuddin, K. Ranjan, R. Sharma, V. Sharma

\section{Saha Institute of Nuclear Physics, HBNI, Kolkata, India}

R. Bhardwaj, R. Bhattacharya, S. Bhattacharya, S. Dey, S. Dutt, S. Dutta, S. Ghosh, N. Majumdar, A. Modak, K. Mondal, S. Mukhopadhyay, S. Nandan, A. Purohit, A. Roy, D. Roy, S. Roy Chowdhury, S. Sarkar, M. Sharan, S. Thakur

\section{Indian Institute of Technology Madras, Madras, India}

P. K. Behera

Bhabha Atomic Research Centre, Mumbai, India

R. Chudasama, D. Dutta, V. Jha, V. Kumar, A. K. Mohanty ${ }^{16}$, P. K. Netrakanti, L. M. Pant, P. Shukla, A. Topkar

Tata Institute of Fundamental Research-A, Mumbai, India

T. Aziz, S. Dugad, B. Mahakud, S. Mitra, G. B. Mohanty, B. Parida, N. Sur, B. Sutar

\section{Tata Institute of Fundamental Research-B, Mumbai, India}

S. Banerjee, S. Bhattacharya, S. Chatterjee, P. Das, M. Guchait, Sa. Jain, S. Kumar, M. Maity ${ }^{25}$, G. Majumder, K. Mazumdar, T. Sarkar ${ }^{25}$, N. Wickramage ${ }^{26}$

Indian Institute of Science Education and Research (IISER), Pune, India

S. Chauhan, S. Dube, V. Hegde, A. Kapoor, K. Kothekar, S. Pandey, A. Rane, S. Sharma

\section{Institute for Research in Fundamental Sciences (IPM), Tehran, Iran}

S. Chenarani ${ }^{27}$, E. Eskandari Tadavani, S. M. Etesami ${ }^{27}$, M. Khakzad, M. Mohammadi Najafabadi, M. Naseri, S. Paktinat Mehdiabadi ${ }^{28}$, F. Rezaei Hosseinabadi, B. Safarzadeh ${ }^{29}$, M. Zeinali

\section{University College Dublin, Dublin, Ireland}

M. Felcini, M. Grunewald

INFN Sezione di Bari ${ }^{a}$, Università di Bari ${ }^{b}$, Politecnico di Bari ${ }^{c}$, Bari, Italy

M. Abbrescia ${ }^{a, b}$, C. Calabria ${ }^{a, b}$, C. Caputo $^{a, b}$, A. Colaleo $^{a}$, D. Creanza $^{a, c}$, L. Cristella $^{a, b}$, N. De Filippis ${ }^{a, c}$,

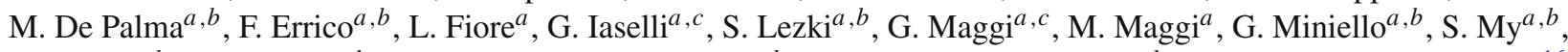

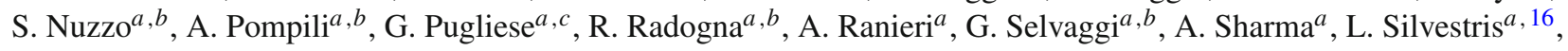
R. Venditti ${ }^{a}$, P. Verwilligen ${ }^{a}$

INFN Sezione di Bologna ${ }^{a}$, Università di Bologna ${ }^{b}$, Bologna, Italy

G. Abbiendi ${ }^{a}$, C. Battilana ${ }^{a, b}$, D. Bonacorsi ${ }^{a}, b$, S. Braibant-Giacomelli ${ }^{a, b}$, R. Campanini $^{a, b}$, P. Capiluppi $^{a, b}$, A. Castro $^{a, b}$, F. R. Cavallo ${ }^{a}$, S. S. Chhibra ${ }^{a}$, G. Codispoti ${ }^{a}, b$, M. Cuffiani ${ }^{a, b}$, G. M. Dallavalle ${ }^{a}$, F. Fabbri ${ }^{a}$, A. Fanfani ${ }^{a}, b$,

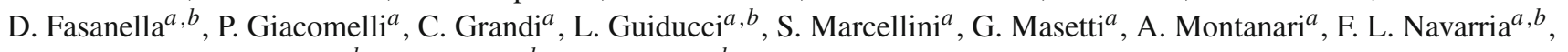
A. Perrotta ${ }^{a}$, A. M. Rossi ${ }^{a, b}$, T. Rovelli ${ }^{a, b}$, G. P. Siroli ${ }^{a}, b$, N. Tosi ${ }^{a}$

INFN Sezione di Catania ${ }^{a}$, Università di Catania ${ }^{b}$, Catania, Italy

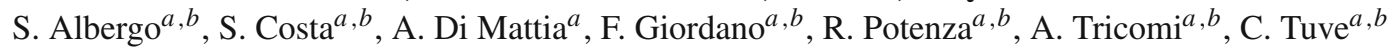

INFN Sezione di Firenze ${ }^{a}$, Università di Firenze ${ }^{b}$, Florence, Italy

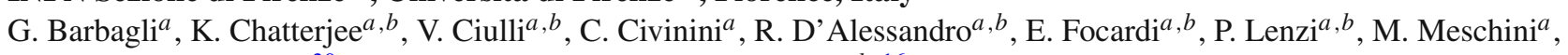
S. Paoletti ${ }^{a}$, L. Russo ${ }^{a}, 30$, G. Sguazzoni ${ }^{a}$, D. Strom ${ }^{a}$, L. Viliani $^{a, b}, 16$

INFN Laboratori Nazionali di Frascati, Frascati, Italy

L. Benussi, S. Bianco, F. Fabbri, D. Piccolo, F. Primavera ${ }^{16}$

INFN Sezione di Genova ${ }^{a}$, Università di Genova ${ }^{b}$, Genoa, Italy

V. Calvelli ${ }^{a, b}$, F. Ferro $^{a}$, E. Robutti ${ }^{a}$, S. Tosi ${ }^{a, b}$

INFN Sezione di Milano-Bicocca ${ }^{a}$, Università di Milano-Bicocca ${ }^{b}$, Milan, Italy

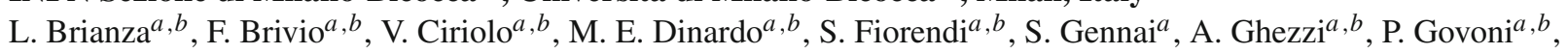

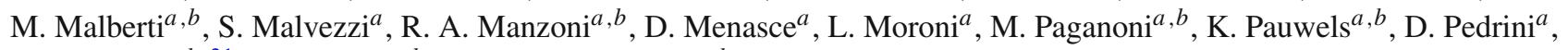
S. Pigazzini ${ }^{a}, b, 31$, S. Ragazzi ${ }^{a, b}$, T. Tabarelli de Fatis ${ }^{a, b}$ 
INFN Sezione di Napoli ${ }^{a}$, Università di Napoli 'Federico II' ${ }^{b}$, Napoli, Italy, Università della Basilicata ${ }^{c}$, Potenza, Italy , Università G. Marconi ${ }^{d}$, Rome, Italy

S. Buontempo ${ }^{a}$, N. Cavallo ${ }^{a, c}$, S. Di Guida ${ }^{a, d}, 16$, F. Fabozzi ${ }^{a, c}$, F. Fienga ${ }^{a, b}$, A. O. M. Iorio ${ }^{a, b}$, W. A. Khan ${ }^{a}$, L. Lista ${ }^{a}$,

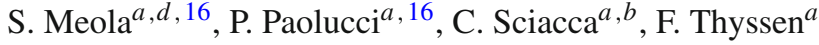

INFN Sezione di Padova ${ }^{a}$, Università di Padova ${ }^{b}$, Padova, Italy, Università di Trento ${ }^{c}$, Trento, Italy P. Azzi ${ }^{a, 16}$, N. Bacchetta ${ }^{a}$, L. Benato ${ }^{a, b}$, D. Bisello ${ }^{a, b}$, A. Boletti $^{a, b}$, R. Carlin ${ }^{a, b}$, A. Carvalho Antunes De Oliveira ${ }^{a, b}$, P. Checchia ${ }^{a}$, P. De Castro Manzano ${ }^{a}$, T. Dorigo ${ }^{a}$, U. Dosselli ${ }^{a}$, F. Gasparini ${ }^{a, b}$, U. Gasparini $^{a}, b$, A. Gozzelino ${ }^{a}$,

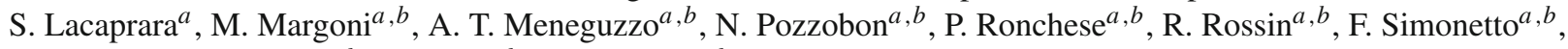
E. Torassa ${ }^{a}$, M. Zanetti ${ }^{a, b}$, P. Zotto ${ }^{a, b}$, G. Zumerle ${ }^{a, b}$

INFN Sezione di Pavia ${ }^{a}$, Università di Pavia ${ }^{b}$, Pavia, Italy

A. Braghieri ${ }^{a}$, F. Fallavollita ${ }^{a, b}$, A. Magnani ${ }^{a, b}$, P. Montagna ${ }^{a}, b$, S. P. Ratti ${ }^{a, b}$, V. Re $^{a}$, M. Ressegotti, C. Riccardi ${ }^{a, b}$, P. Salvini ${ }^{a}$, I. Vai ${ }^{a, b}$, P. Vitulo ${ }^{a, b}$

INFN Sezione di Perugia ${ }^{a}$, Università di Perugia ${ }^{b}$, Perugia, Italy

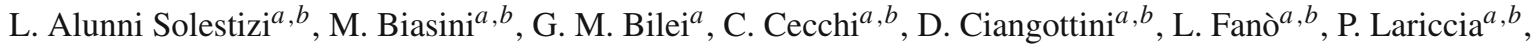

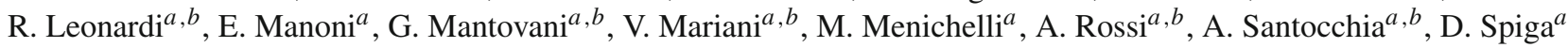

INFN Sezione di Pisa ${ }^{a}$, Università di Pisa ${ }^{b}$, Scuola Normale Superiore di Pisa ${ }^{c}$, Pisa, Italy

K. Androsov ${ }^{a}$, P. Azzurri ${ }^{a}, 16$, G. Bagliesi ${ }^{a}$, J. Bernardini $^{a}$, T. Boccali ${ }^{a}$, L. Borrello, R. Castaldi ${ }^{a}$, M. A. Ciocci ${ }^{a}, b$,

R. Dell'Orso ${ }^{a}$, G. Fedi ${ }^{a}$, L. Giannini ${ }^{a, c}$, A. Giassi ${ }^{a}$, M. T. Grippo ${ }^{a}, 30$, F. Ligabue $^{a, c}$, T. Lomtadze ${ }^{a}$, E. Manca ${ }^{a, c}$,

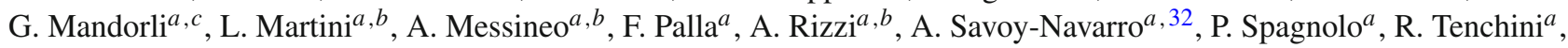

G. Tonelli ${ }^{a, b}$, A. Venturi ${ }^{a}$, P. G. Verdini ${ }^{a}$

INFN Sezione di $\operatorname{Roma}^{a}$, Sapienza Università di Roma ${ }^{b}$, Rome, Italy

L. Barone ${ }^{a, b}$, F. Cavallari ${ }^{a}$, M. Cipriani ${ }^{a, b}$, D. Del Re ${ }^{a, b, 16}$, M. Diemoz ${ }^{a}$, S. Gelli ${ }^{a, b}$, E. Longo ${ }^{a, b}$, F. Margaroli $^{a, b}$,

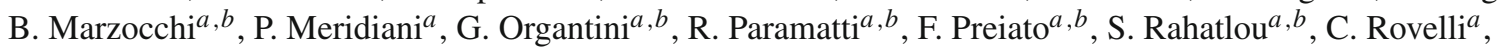

F. Santanastasio ${ }^{a, b}$

INFN Sezione di Torino ${ }^{a}$, Università di Torino ${ }^{b}$, Torino, Italy, Università del Piemonte Orientale ${ }^{c}$, Novara, Italy

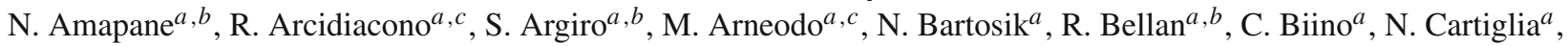

F. Cenna ${ }^{a, b}$, M. Costa ${ }^{a, b}$, R. Covarelli ${ }^{a, b}$, A. Degano ${ }^{a, b}$, N. Demaria ${ }^{a}$, B. Kiani ${ }^{a, b}$, C. Mariotti ${ }^{a}$, S. Maselli ${ }^{a}$,

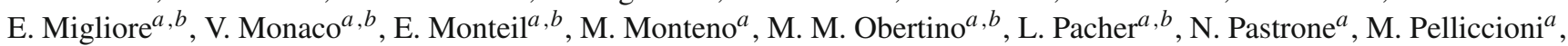

G. L. Pinna Angioni ${ }^{a, b}$, F. Ravera ${ }^{a, b}$, A. Romero ${ }^{a, b}$, M. Ruspa $^{a, c}$, R. Sacchi $^{a, b}$, K. Shchelina $^{a, b}$, V. Sola ${ }^{a}$, A. Solano ${ }^{a, b}$,

A. Staiano ${ }^{a}$, P. $\operatorname{Traczyk}^{a, b}$

INFN Sezione di Trieste ${ }^{a}$, Università di Trieste ${ }^{b}$, Trieste, Italy

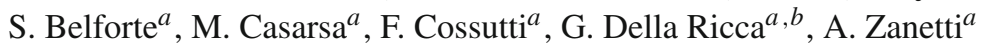

Kyungpook National University, Daegu, Korea

D. H. Kim, G. N. Kim, M. S. Kim, J. Lee, S. Lee, S. W. Lee, C. S. Moon, Y. D. Oh, S. Sekmen, D. C. Son, Y. C. Yang

Chonbuk National University, Jeonju, Korea

A. Lee

Institute for Universe and Elementary Particles, Chonnam National University, Kwangju, Korea

H. Kim, D. H. Moon, G. Oh

Hanyang University, Seoul, Korea

J. A. Brochero Cifuentes, J. Goh, T. J. Kim

Korea University, Seoul, Korea

S. Cho, S. Choi, Y. Go, D. Gyun, S. Ha, B. Hong, Y. Jo, Y. Kim, K. Lee, K. S. Lee, S. Lee, J. Lim, S. K. Park, Y. Roh

Seoul National University, Seoul, Korea

J. Almond, J. Kim, J. S. Kim, H. Lee, K. Lee, K. Nam, S. B. Oh, B. C. Radburn-Smith, S. h. Seo, U. K. Yang, H. D. Yoo,

G. B. Yu 
University of Seoul, Seoul, Korea

M. Choi, H. Kim, J. H. Kim, J. S. H. Lee, I. C. Park, G. Ryu

Sungkyunkwan University, Suwon, Korea

Y. Choi, C. Hwang, J. Lee, I. Yu

Vilnius University, Vilnius, Lithuania

V. Dudenas, A. Juodagalvis, J. Vaitkus

National Centre for Particle Physics, Universiti Malaya, Kuala Lumpur, Malaysia

I. Ahmed, Z. A. Ibrahim, M. A. B. Md Ali ${ }^{33}$, F. Mohamad Idris ${ }^{34}$, W. A. T. Wan Abdullah, M. N. Yusli, Z. Zolkapli

Centro de Investigacion y de Estudios Avanzados del IPN, Mexico City, Mexico

H. Castilla-Valdez, E. De La Cruz-Burelo, I. Heredia-De La Cruz ${ }^{35}$, R. Lopez-Fernandez, J. Mejia Guisao,

A. Sanchez-Hernandez

Universidad Iberoamericana, Mexico City, Mexico

S. Carrillo Moreno, C. Oropeza Barrera, F. Vazquez Valencia

Benemerita Universidad Autonoma de Puebla, Puebla, Mexico

I. Pedraza, H. A. Salazar Ibarguen, C. Uribe Estrada

Universidad Autónoma de San Luis Potosí, San Luis Potosí, Mexico

A. Morelos Pineda

University of Auckland, Auckland, New Zealand

D. Krofcheck

University of Canterbury, Christchurch, New Zealand

P. H. Butler

National Centre for Physics, Quaid-I-Azam University, Islamabad, Pakistan

A. Ahmad, M. Ahmad, Q. Hassan, H. R. Hoorani, A. Saddique, M. A. Shah, M. Shoaib, M. Waqas

National Centre for Nuclear Research, Swierk, Poland

H. Bialkowska, M. Bluj, B. Boimska, T. Frueboes, M. Górski, M. Kazana, K. Nawrocki, K. Romanowska-Rybinska,

M. Szleper, P. Zalewski

Faculty of Physics, Institute of Experimental Physics, University of Warsaw, Warsaw, Poland

K. Bunkowski, A. Byszuk ${ }^{36}$, K. Doroba, A. Kalinowski, M. Konecki, J. Krolikowski, M. Misiura, M. Olszewski,

A. Pyskir, M. Walczak

Laboratório de Instrumentação e Física Experimental de Partículas, Lisbon, Portugal

P. Bargassa, C. Beirão Da Cruz E Silva, B. Calpas, A. Di Francesco, P. Faccioli, M. Gallinaro, J. Hollar, N. Leonardo,

L. Lloret Iglesias, M. V. Nemallapudi, J. Seixas, O. Toldaiev, D. Vadruccio, J. Varela

Joint Institute for Nuclear Research, Dubna, Russia

S. Afanasiev, P. Bunin, M. Gavrilenko, I. Golutvin, I. Gorbunov, A. Kamenev, V. Karjavin, A. Lanev, A. Malakhov,

V. Matveev ${ }^{37,38}$, V. Palichik, V. Perelygin, S. Shmatov, S. Shulha, N. Skatchkov, V. Smirnov, N. Voytishin, A. Zarubin

Petersburg Nuclear Physics Institute, Gatchina, St. Petersburg, Russia

Y. Ivanov, V. Kim ${ }^{39}$, E. Kuznetsova ${ }^{40}$, P. Levchenko, V. Murzin, V. Oreshkin, I. Smirnov, V. Sulimov, L. Uvarov,

S. Vavilov, A. Vorobyev

Institute for Nuclear Research, Moscow, Russia

Yu. Andreev, A. Dermenev, S. Gninenko, N. Golubev, A. Karneyeu, M. Kirsanov, N. Krasnikov, A. Pashenkov, D. Tlisov,

A. Toropin

Institute for Theoretical and Experimental Physics, Moscow, Russia

V. Epshteyn, V. Gavrilov, N. Lychkovskaya, V. Popov, I. Pozdnyakov, G. Safronov, A. Spiridonov, A. Stepennov, M. Toms, E. Vlasov, A. Zhokin 
Moscow Institute of Physics and Technology, Moscow, Russia T. Aushev, A. Bylinkin ${ }^{38}$

National Research Nuclear University 'Moscow Engineering Physics Institute' (MEPhI), Moscow, Russia R. Chistov ${ }^{41}$, M. Danilov ${ }^{41}$, P. Parygin, D. Philippov, S. Polikarpov, E. Tarkovskii

P.N. Lebedev Physical Institute, Moscow, Russia

V. Andreev, M. Azarkin ${ }^{38}$, I. Dremin ${ }^{38}$, M. Kirakosyan ${ }^{38}$, A. Terkulov

Skobeltsyn Institute of Nuclear Physics, Lomonosov Moscow State University, Moscow, Russia

A. Baskakov, A. Belyaev, E. Boos, M. Dubinin ${ }^{42}$, L. Dudko, A. Ershov, A. Gribushin, V. Klyukhin, O. Kodolova, I. Lokhtin, I. Miagkov, S. Obraztsov, S. Petrushanko, V. Savrin, A. Snigirev

Novosibirsk State University (NSU), Novosibirsk, Russia

V. Blinov ${ }^{43}$, Y. Skovpen ${ }^{43}$, D. Shtol ${ }^{43}$

State Research Center of Russian Federation, Institute for High Energy Physics, Protvino, Russia

I. Azhgirey, I. Bayshev, S. Bitioukov, D. Elumakhov, V. Kachanov, A. Kalinin, D. Konstantinov, V. Krychkine, V. Petrov, R. Ryutin, A. Sobol, S. Troshin, N. Tyurin, A. Uzunian, A. Volkov

Faculty of Physics and Vinca Institute of Nuclear Sciences, University of Belgrade, Belgrade, Serbia P. Adzic ${ }^{44}$, P. Cirkovic, D. Devetak, M. Dordevic, J. Milosevic, V. Rekovic

Centro de Investigaciones Energéticas Medioambientales y Tecnológicas (CIEMAT), Madrid, Spain

J. Alcaraz Maestre, M. Barrio Luna, M. Cerrada, N. Colino, B. De La Cruz, A. Delgado Peris, A. Escalante Del Valle,

C. Fernandez Bedoya, J. P. Fernández Ramos, J. Flix, M. C. Fouz, P. Garcia-Abia, O. Gonzalez Lopez, S. Goy Lopez, J.

M. Hernandez, M. I. Josa, A. Pérez-Calero Yzquierdo, J. Puerta Pelayo, A. Quintario Olmeda, I. Redondo, L. Romero, M.

S. Soares, A. Álvarez Fernández

Universidad Autónoma de Madrid, Madrid, Spain

J. F. de Trocóniz, M. Missiroli, D. Moran

Universidad de Oviedo, Oviedo, Spain

J. Cuevas, C. Erice, J. Fernandez Menendez, I. Gonzalez Caballero, J. R. González Fernández, E. Palencia Cortezon,

S. Sanchez Cruz, I. Suárez Andrés, P. Vischia, J. M. Vizan Garcia

Instituto de Física de Cantabria (IFCA), CSIC-Universidad de Cantabria, Santander, Spain

I. J. Cabrillo, A. Calderon, B. Chazin Quero, E. Curras, M. Fernandez, J. Garcia-Ferrero, G. Gomez, A. Lopez Virto,

J. Marco, C. Martinez Rivero, P. Martinez Ruiz del Arbol, F. Matorras, J. Piedra Gomez, T. Rodrigo, A. Ruiz-Jimeno,

L. Scodellaro, N. Trevisani, I. Vila, R. Vilar Cortabitarte

CERN, European Organization for Nuclear Research, Geneva, Switzerland

D. Abbaneo, E. Auffray, P. Baillon, A. H. Ball, D. Barney, M. Bianco, P. Bloch, A. Bocci, C. Botta, T. Camporesi,

R. Castello, M. Cepeda, G. Cerminara, E. Chapon, Y. Chen, D. d'Enterria, A. Dabrowski, V. Daponte, A. David,

M. De Gruttola, A. De Roeck, E. Di Marco ${ }^{45}$, M. Dobson, B. Dorney, T. du Pree, M. Dünser, N. Dupont, A. Elliott-Peisert,

P. Everaerts, G. Franzoni, J. Fulcher, W. Funk, D. Gigi, K. Gill, F. Glege, D. Gulhan, S. Gundacker, M. Guthoff, P. Harris,

J. Hegeman, V. Innocente, P. Janot, O. Karacheban ${ }^{19}$, J. Kieseler, H. Kirschenmann, V. Knünz, A. Kornmayer ${ }^{16}$, M.

J. Kortelainen, C. Lange, P. Lecoq, C. Lourenço, M. T. Lucchini, L. Malgeri, M. Mannelli, A. Martelli, F. Meijers, J.

A. Merlin, S. Mersi, E. Meschi, P. Milenovic ${ }^{46}$, F. Moortgat, M. Mulders, H. Neugebauer, S. Orfanelli, L. Orsini, L. Pape,

E. Perez, M. Peruzzi, A. Petrilli, G. Petrucciani, A. Pfeiffer, M. Pierini, A. Racz, T. Reis, G. Rolandi ${ }^{47}$, M. Rovere,

H. Sakulin, C. Schäfer, C. Schwick, M. Seidel, M. Selvaggi, A. Sharma, P. Silva, P. Sphicas ${ }^{48}$, J. Steggemann, M. Stoye,

M. Tosi, D. Treille, A. Triossi, A. Tsirou, V. Veckalns ${ }^{49}$, G. I. Veres ${ }^{21}$, M. Verweij, N. Wardle, W. D. Zeuner

Paul Scherrer Institut, Villigen, Switzerland

W. Bertl ${ }^{\dagger}$, L. Caminada ${ }^{50}$, K. Deiters, W. Erdmann, R. Horisberger, Q. Ingram, H. C. Kaestli, D. Kotlinski,

U. Langenegger, T. Rohe, S. A. Wiederkehr

Institute for Particle Physics ETH Zurich, Zurich, Switzerland

F. Bachmair, L. Bäni, P. Berger, L. Bianchini, B. Casal, G. Dissertori, M. Dittmar, M. Donegà, C. Grab, C. Heidegger, 
D. Hits, J. Hoss, G. Kasieczka, T. Klijnsma, W. Lustermann, B. Mangano, M. Marionneau, M. T. Meinhard, D. Meister, F. Micheli, P. Musella, F. Nessi-Tedaldi, F. Pandolfi, J. Pata, F. Pauss, G. Perrin, L. Perrozzi, M. Quittnat, M. Schönenberger, L. Shchutska, V. R. Tavolaro, K. Theofilatos, M. L. Vesterbacka Olsson, R. Wallny, A. Zagozdzinska ${ }^{36}$, D. H. Zhu

\section{Universität Zürich, Zurich, Switzerland}

T. K. Aarrestad, C. Amsler ${ }^{51}$, M. F. Canelli, A. De Cosa, S. Donato, C. Galloni, T. Hreus, B. Kilminster, J. Ngadiuba,

D. Pinna, G. Rauco, P. Robmann, D. Salerno, C. Seitz, A. Zucchetta

\section{National Central University, Chung-Li, Taiwan}

V. Candelise, T. H. Doan, Sh. Jain, R. Khurana, C. M. Kuo, W. Lin, A. Pozdnyakov, S. S. Yu

\section{National Taiwan University (NTU), Taipei, Taiwan}

Arun Kumar, P. Chang, Y. Chao, K. F. Chen, P. H. Chen, F. Fiori, W.-S. Hou, Y. Hsiung, Y. F. Liu, R.-S. Lu,

M. Miñano Moya, E. Paganis, A. Psallidas, J. f. Tsai

\section{Department of Physics, Faculty of Science, Chulalongkorn University, Bangkok, Thailand}

B. Asavapibhop, K. Kovitanggoon, G. Singh, N. Srimanobhas

Physics Department, Science and Art Faculty, Cukurova University, Adana, Turkey

A. Adiguzel ${ }^{52}$, F. Boran, S. Cerci ${ }^{53}$, S. Damarseckin, Z. S. Demiroglu, C. Dozen, I. Dumanoglu, S. Girgis, G. Gokbulut, Y. Guler, I. $\mathrm{Hos}^{54}$, E. E. Kangal ${ }^{55}$, O. Kara, A. Kayis Topaksu, U. Kiminsu, M. Oglakci, G. Onengut ${ }^{56}$, K. Ozdemir ${ }^{57}$, D. Sunar Cerci ${ }^{53}$, B. Tali ${ }^{53}$, S. Turkcapar, I. S. Zorbakir, C. Zorbilmez

Physics Department, Middle East Technical University, Ankara, Turkey

B. Bilin, G. Karapinar ${ }^{58}$, K. Ocalan ${ }^{59}$, M. Yalvac, M. Zeyrek

Bogazici University, Istanbul, Turkey

E. Gülmez, M. Kaya ${ }^{60}$, O. Kaya ${ }^{61}$, S. Tekten, E. A. Yetkin ${ }^{62}$

Istanbul Technical University, Istanbul, Turkey

M. N. Agaras, S. Atay, A. Cakir, K. Cankocak

Institute for Scintillation Materials of National Academy of Science of Ukraine, Kharkov, Ukraine

B. Grynyov

National Scientific Center, Kharkov Institute of Physics and Technology, Kharkov, Ukraine

L. Levchuk, P. Sorokin

University of Bristol, Bristol, United Kingdom

R. Aggleton, F. Ball, L. Beck, J. J. Brooke, D. Burns, E. Clement, D. Cussans, O. Davignon, H. Flacher, J. Goldstein, M. Grimes, G. P. Heath, H. F. Heath, J. Jacob, L. Kreczko, C. Lucas, D. M. Newbold ${ }^{63}$, S. Paramesvaran, A. Poll, T. Sakuma, S. Seif El Nasr-storey, D. Smith, V. J. Smith

\section{Rutherford Appleton Laboratory, Didcot, United Kingdom}

K. W. Bell, A. Belyaev ${ }^{64}$, C. Brew, R. M. Brown, L. Calligaris, D. Cieri, D. J. A. Cockerill, J. A. Coughlan, K. Harder, S. Harper, E. Olaiya, D. Petyt, C. H. Shepherd-Themistocleous, A. Thea, I. R. Tomalin, T. Williams

\section{Imperial College, London, UK}

R. Bainbridge, S. Breeze, O. Buchmuller, A. Bundock, S. Casasso, M. Citron, D. Colling, L. Corpe, P. Dauncey,

G. Davies, A. De Wit, M. Della Negra, R. Di Maria, A. Elwood, Y. Haddad, G. Hall, G. Iles, T. James, R. Lane, C. Laner, L. Lyons, A.-M. Magnan, S. Malik, L. Mastrolorenzo, T. Matsushita, J. Nash, A. Nikitenko ${ }^{6}$, V. Palladino, M. Pesaresi, D. M. Raymond, A. Richards, A. Rose, E. Scott, C. Seez, A. Shtipliyski, S. Summers, A. Tapper, K. Uchida,

M. Vazquez Acosta ${ }^{65}$, T. Virdee ${ }^{16}$, D. Winterbottom, J. Wright, S. C. Zenz

Brunel University, Uxbridge, UK

J. E. Cole, P. R. Hobson, A. Khan, P. Kyberd, I. D. Reid, P. Symonds, L. Teodorescu, M. Turner

Baylor University, Waco, USA

A. Borzou, K. Call, J. Dittmann, K. Hatakeyama, H. Liu, N. Pastika, C. Smith 


\section{Catholic University of America, Washington, USA}

R. Bartek, A. Dominguez

The University of Alabama, Tuscaloosa, USA

A. Buccilli, S. I. Cooper, C. Henderson, P. Rumerio, C. West

Boston University, Boston, USA

D. Arcaro, A. Avetisyan, T. Bose, D. Gastler, D. Rankin, C. Richardson, J. Rohlf, L. Sulak, D. Zou

\section{Brown University, Providence, USA}

G. Benelli, D. Cutts, A. Garabedian, J. Hakala, U. Heintz, J. M. Hogan, K. H. M. Kwok, E. Laird, G. Landsberg, Z. Mao, M. Narain, J. Pazzini, S. Piperov, S. Sagir, R. Syarif, D. Yu

\section{University of California Davis, Davis, USA}

R. Band, C. Brainerd, D. Burns, M. Calderon De La Sanchez, M. Chertok, J. Conway, R. Conway, P. T. Cox, R. Erbacher,

C. Flores, G. Funk, M. Gardner, W. Ko, R. Lander, C. Mclean, M. Mulhearn, D. Pellett, J. Pilot, S. Shalhout, M. Shi,

J. Smith, M. Squires, D. Stolp, K. Tos, M. Tripathi, Z. Wang

University of California, Los Angeles, USA

M. Bachtis, C. Bravo, R. Cousins, A. Dasgupta, A. Florent, J. Hauser, M. Ignatenko, N. Mccoll, D. Saltzberg,

C. Schnaible, V. Valuev

University of California Riverside, Riverside, USA

E. Bouvier, K. Burt, R. Clare, J. Ellison, J. W. Gary, S. M. A. Ghiasi Shirazi, G. Hanson, J. Heilman, P. Jandir, E. Kennedy, F. Lacroix, O. R. Long, M. Olmedo Negrete, M. I. Paneva, A. Shrinivas, W. Si, L. Wang, H. Wei, S. Wimpenny, B. R. Yates

University of California San Diego, La Jolla, USA

J. G. Branson, S. Cittolin, M. Derdzinski, B. Hashemi, A. Holzner, D. Klein, G. Kole, V. Krutelyov, J. Letts, I. Macneill, M. Masciovecchio, D. Olivito, S. Padhi, M. Pieri, M. Sani, V. Sharma, S. Simon, M. Tadel, A. Vartak, S. Wasserbaech ${ }^{66}$, J. Wood, F. Würthwein, A. Yagil, G. Zevi Della Porta

\section{Department of Physics, University of California Santa Barbara, Santa Barbara, USA}

N. Amin, R. Bhandari, J. Bradmiller-Feld, C. Campagnari, A. Dishaw, V. Dutta, M. Franco Sevilla, C. George, F. Golf,

L. Gouskos, J. Gran, R. Heller, J. Incandela, S. D. Mullin, A. Ovcharova, H. Qu, J. Richman, D. Stuart, I. Suarez, J. Yoo

California Institute of Technology, Pasadena, USA

D. Anderson, J. Ben david, A. Bornheim, J. M. Lawhorn, H. B. Newman, T. Nguyen, C. Pena, M. Spiropulu, J. R. Vlimant,

S. Xie, Z. Zhang, R. Y. Zhu

\section{Carnegie Mellon University, Pittsburgh, USA}

M. B. Andrews, T. Ferguson, T. Mudholkar, M. Paulini, J. Russ, M. Sun, H. Vogel, I. Vorobiev, M. Weinberg

\section{University of Colorado Boulder, Boulder, USA}

J. P. Cumalat, W. T. Ford, F. Jensen, A. Johnson, M. Krohn, S. Leontsinis, T. Mulholland, K. Stenson, S. R. Wagner

\section{Cornell University, Ithaca, USA}

J. Alexander, J. Chaves, J. Chu, S. Dittmer, K. Mcdermott, N. Mirman, J. R. Patterson, A. Rinkevicius, A. Ryd,

L. Skinnari, L. Soffi, S. M. Tan, Z. Tao, J. Thom, J. Tucker, P. Wittich, M. Zientek

\section{Fermi National Accelerator Laboratory, Batavia, USA}

S. Abdullin, M. Albrow, G. Apollinari, A. Apresyan, A. Apyan, S. Banerjee, L. A. T. Bauerdick, A. Beretvas, J. Berryhill, P. C. Bhat, G. Bolla, K. Burkett, J. N. Butler, A. Canepa, G. B. Cerati, H. W. K. Cheung, F. Chlebana, M. Cremonesi,

J. Duarte, V. D. Elvira, J. Freeman, Z. Gecse, E. Gottschalk, L. Gray, D. Green, S. Grünendahl, O. Gutsche, R. M. Harris,

S. Hasegawa, J. Hirschauer, Z. Hu, B. Jayatilaka, S. Jindariani, M. Johnson, U. Joshi, B. Klima, B. Kreis, S. Lammel,

D. Lincoln, R. Lipton, M. Liu, T. Liu, R. Lopes De Sá, J. Lykken, K. Maeshima, N. Magini, J. M. Marraffino,

S. Maruyama, D. Mason, P. McBride, P. Merkel, S. Mrenna, S. Nahn, V. O’Dell, K. Pedro, O. Prokofyev, G. Rakness,

L. Ristori, B. Schneider, E. Sexton-Kennedy, A. Soha, W. J. Spalding, L. Spiegel, S. Stoynev, J. Strait, N. Strobbe,

L. Taylor, S. Tkaczyk, N. V. Tran, L. Uplegger, E. W. Vaandering, C. Vernieri, M. Verzocchi, R. Vidal, M. Wang, H.

A. Weber, A. Whitbeck 
University of Florida, Gainesville, USA

D. Acosta, P. Avery, P. Bortignon, D. Bourilkov, A. Brinkerhoff, A. Carnes, M. Carver, D. Curry, S. Das, R. D. Field, I.

K. Furic, J. Konigsberg, A. Korytov, K. Kotov, P. Ma, K. Matchev, H. Mei, G. Mitselmakher, D. Rank, D. Sperka,

N. Terentyev, L. Thomas, J. Wang, S. Wang, J. Yelton

Florida International University, Miami, USA

Y. R. Joshi, S. Linn, P. Markowitz, J. L. Rodriguez

Florida State University, Tallahassee, USA

A. Ackert, T. Adams, A. Askew, S. Hagopian, V. Hagopian, K. F. Johnson, T. Kolberg, G. Martinez, T. Perry, H. Prosper, A. Saha, A. Santra, R. Yohay

Florida Institute of Technology, Melbourne, USA

M. M. Baarmand, V. Bhopatkar, S. Colafranceschi, M. Hohlmann, D. Noonan, T. Roy, F. Yumiceva

University of Illinois at Chicago (UIC), Chicago, USA

M. R. Adams, L. Apanasevich, D. Berry, R. R. Betts, R. Cavanaugh, X. Chen, O. Evdokimov, C. E. Gerber, D. A. Hangal, D. J. Hofman, K. Jung, J. Kamin, I. D. Sandoval Gonzalez, M. B. Tonjes, H. Trauger, N. Varelas, H. Wang, Z. Wu, J. Zhang

The University of Iowa, Iowa City, USA

B. Bilki ${ }^{67}$, W. Clarida, K. Dilsiz ${ }^{68}$, S. Durgut, R. P. Gandrajula, M. Haytmyradov, V. Khristenko, J.-P. Merlo, H. Mermerkaya ${ }^{69}$, A. Mestvirishvili, A. Moeller, J. Nachtman, H. Ogul ${ }^{70}$, Y. Onel, F. Ozok ${ }^{71}$, A. Penzo, C. Snyder, E. Tiras, J. Wetzel, K. Yi

Johns Hopkins University, Baltimore, USA

B. Blumenfeld, A. Cocoros, N. Eminizer, D. Fehling, L. Feng, A. V. Gritsan, P. Maksimovic, J. Roskes, U. Sarica, M. Swartz, M. Xiao, C. You

The University of Kansas, Lawrence, USA

A. Al-bataineh, P. Baringer, A. Bean, S. Boren, J. Bowen, J. Castle, S. Khalil, A. Kropivnitskaya, D. Majumder, W. Mcbrayer, M. Murray, C. Royon, S. Sanders, E. Schmitz, R. Stringer, J. D. Tapia Takaki, Q. Wang

Kansas State University, Manhattan, USA

A. Ivanov, K. Kaadze, Y. Maravin, A. Mohammadi, L. K. Saini, N. Skhirtladze, S. Toda

Lawrence Livermore National Laboratory, Livermore, USA

F. Rebassoo, D. Wright

University of Maryland, College Park, USA

C. Anelli, A. Baden, O. Baron, A. Belloni, B. Calvert, S. C. Eno, C. Ferraioli, N. J. Hadley, S. Jabeen, G. Y. Jeng, R.

G. Kellogg, J. Kunkle, A. C. Mignerey, F. Ricci-Tam, Y. H. Shin, A. Skuja, S. C. Tonwar

Massachusetts Institute of Technology, Cambridge, USA

D. Abercrombie, B. Allen, V. Azzolini, R. Barbieri, A. Baty, R. Bi, S. Brandt, W. Busza, I. A. Cali, M. D’Alfonso, Z. Demiragli, G. Gomez Ceballos, M. Goncharov, D. Hsu, Y. Iiyama, G. M. Innocenti, M. Klute, D. Kovalskyi, Y. S. Lai, Y.-J. Lee, A. Levin, P. D. Luckey, B. Maier, A. C. Marini, C. Mcginn, C. Mironov, S. Narayanan, X. Niu, C. Paus,

C. Roland, G. Roland, J. Salfeld-Nebgen, G. S. F. Stephans, K. Tatar, D. Velicanu, J. Wang, T. W. Wang, B. Wyslouch

University of Minnesota, Minneapolis, USA

A. C. Benvenuti, R. M. Chatterjee, A. Evans, P. Hansen, S. Kalafut, Y. Kubota, Z. Lesko, J. Mans, S. Nourbakhsh, N. Ruckstuhl, R. Rusack, J. Turkewitz

\section{University of Mississippi, Oxford, USA}

J. G. Acosta, S. Oliveros

\section{University of Nebraska-Lincoln, Lincoln, USA}

E. Avdeeva, K. Bloom, D. R. Claes, C. Fangmeier, R. Gonzalez Suarez, R. Kamalieddin, I. Kravchenko, J. Monroy, J.

E. Siado, G. R. Snow, B. Stieger 
State University of New York at Buffalo, Buffalo, USA

M. Alyari, J. Dolen, A. Godshalk, C. Harrington, I. Iashvili, D. Nguyen, A. Parker, S. Rappoccio, B. Roozbahani

Northeastern University, Boston, USA

G. Alverson, E. Barberis, A. Hortiangtham, A. Massironi, D. M. Morse, D. Nash, T. Orimoto, R. Teixeira De Lima,

D. Trocino, D. Wood

Northwestern University, Evanston, USA

S. Bhattacharya, O. Charaf, K. A. Hahn, N. Mucia, N. Odell, B. Pollack, M. H. Schmitt, K. Sung, M. Trovato, M. Velasco

University of Notre Dame, Notre Dame, USA

N. Dev, M. Hildreth, K. Hurtado Anampa, C. Jessop, D. J. Karmgard, N. Kellams, K. Lannon, N. Loukas, N. Marinelli, F. Meng, C. Mueller, Y. Musienko ${ }^{37}$, M. Planer, A. Reinsvold, R. Ruchti, G. Smith, S. Taroni, M. Wayne, M. Wolf,

A. Woodard

The Ohio State University, Columbus, USA

J. Alimena, L. Antonelli, B. Bylsma, L. S. Durkin, S. Flowers, B. Francis, A. Hart, C. Hill, W. Ji, B. Liu, W. Luo,

D. Puigh, B. L. Winer, H. W. Wulsin

\section{Princeton University, Princeton, USA}

A. Benaglia, S. Cooperstein, O. Driga, P. Elmer, J. Hardenbrook, P. Hebda, S. Higginbotham, D. Lange, J. Luo,

D. Marlow, K. Mei, I. Ojalvo, J. Olsen, C. Palmer, P. Piroué, D. Stickland, C. Tully

\section{University of Puerto Rico, Mayaguez, USA}

S. Malik, S. Norberg

Purdue University, West Lafayette, USA

A. Barker, V. E. Barnes, S. Folgueras, L. Gutay, M. K. Jha, M. Jones, A. W. Jung, A. Khatiwada, D. H. Miller,

N. Neumeister, C. C. Peng, J. F. Schulte, J. Sun, F. Wang, W. Xie

Purdue University Northwest, Hammond, USA

T. Cheng, N. Parashar, J. Stupak

Rice University, Houston, USA

A. Adair, B. Akgun, Z. Chen, K. M. Ecklund, F. J. M. Geurts, M. Guilbaud, W. Li, B. Michlin, M. Northup, B. P. Padley, J. Roberts, J. Rorie, Z. Tu, J. Zabel

University of Rochester, Rochester, USA

A. Bodek, P. de Barbaro, R. Demina, Y. t. Duh, T. Ferbel, M. Galanti, A. Garcia-Bellido, J. Han, O. Hindrichs,

A. Khukhunaishvili, K. H. Lo, P. Tan, M. Verzetti

The Rockefeller University, New York, USA

R. Ciesielski, K. Goulianos, C. Mesropian

Rutgers, The State University of New Jersey, Piscataway, USA

A. Agapitos, J. P. Chou, Y. Gershtein, T. A. Gómez Espinosa, E. Halkiadakis, M. Heindl, E. Hughes, S. Kaplan,

R. Kunnawalkam Elayavalli, S. Kyriacou, A. Lath, R. Montalvo, K. Nash, M. Osherson, H. Saka, S. Salur, S. Schnetzer,

D. Sheffield, S. Somalwar, R. Stone, S. Thomas, P. Thomassen, M. Walker

University of Tennessee, Knoxville, USA

A. G. Delannoy, M. Foerster, J. Heideman, G. Riley, K. Rose, S. Spanier, K. Thapa

Texas A\&M University, College Station, USA

O. Bouhali ${ }^{72}$, A. Castaneda Hernandez ${ }^{72}$, A. Celik, M. Dalchenko, M. De Mattia, A. Delgado, S. Dildick, R. Eusebi,

J. Gilmore, T. Huang, T. Kamon ${ }^{73}$, R. Mueller, Y. Pakhotin, R. Patel, A. Perloff, L. Perniè, D. Rathjens, A. Safonov,

A. Tatarinov, K. A. Ulmer

Texas Tech University, Lubbock, USA

N. Akchurin, J. Damgov, F. De Guio, P. R. Dudero, J. Faulkner, E. Gurpinar, S. Kunori, K. Lamichhane, S. W. Lee,

T. Libeiro, T. Peltola, S. Undleeb, I. Volobouev, Z. Wang 


\section{Vanderbilt University, Nashville, USA}

S. Greene, A. Gurrola, R. Janjam, W. Johns, C. Maguire, A. Melo, H. Ni, P. Sheldon, S. Tuo, J. Velkovska, Q. Xu

\section{University of Virginia, Charlottesville, USA}

M. W. Arenton, P. Barria, B. Cox, R. Hirosky, A. Ledovskoy, H. Li, C. Neu, T. Sinthuprasith, X. Sun, Y. Wang, E. Wolfe, F. Xia

\section{Wayne State University, Detroit, USA}

R. Harr, P. E. Karchin, J. Sturdy, S. Zaleski

\section{University of Wisconsin-Madison, Madison, WI, USA}

M. Brodski, J. Buchanan, C. Caillol, S. Dasu, L. Dodd, S. Duric, B. Gomber, M. Grothe, M. Herndon, A. Hervé, U. Hussain, P. Klabbers, A. Lanaro, A. Levine, K. Long, R. Loveless, G. A. Pierro, G. Polese, T. Ruggles, A. Savin, N. Smith, W. H. Smith, D. Taylor, N. Woods

\section{$\dagger$ Deceased}

1: Also at Vienna University of Technology, Vienna, Austria

2: Also at State Key Laboratory of Nuclear Physics and Technology, Peking University, Beijing, China

3: Also at Universidade Estadual de Campinas, Campinas, Brazil

4: Also at Universidade Federal de Pelotas, Pelotas, Brazil

5: Also at Université Libre de Bruxelles, Bruxelles, Belgium

6: Also at Institute for Theoretical and Experimental Physics, Moscow, Russia

7: Also at Joint Institute for Nuclear Research, Dubna, Russia

8: Also at Helwan University, Cairo, Egypt

9: Now at Zewail City of Science and Technology, Zewail, Egypt

10: Now at Fayoum University, El-Fayoum, Egypt

11: Also at British University in Egypt, Cairo, Egypt

12: Now at Ain Shams University, Cairo, Egypt

13: Also at Université de Haute Alsace, Mulhouse, France

14: Also at Skobeltsyn Institute of Nuclear Physics, Lomonosov Moscow State University, Moscow, Russia

15: Also at Tbilisi State University, Tbilisi, Georgia

16: Also at CERN, European Organization for Nuclear Research, Geneva, Switzerland

17: Also at RWTH Aachen University, III. Physikalisches Institut A, Aachen, Germany

18: Also at University of Hamburg, Hamburg, Germany

19: Also at Brandenburg University of Technology, Cottbus, Germany

20: Also at Institute of Nuclear Research ATOMKI, Debrecen, Hungary

21: Also at MTA-ELTE Lendület CMS Particle and Nuclear Physics Group, Eötvös Loránd University, Budapest, Hungary

22: Also at Institute of Physics, University of Debrecen, Debrecen, Hungary

23: Also at Indian Institute of Technology Bhubaneswar, Bhubaneswar, India

24: Also at Institute of Physics, Bhubaneswar, India

25: Also at University of Visva-Bharati, Santiniketan, India

26: Also at University of Ruhuna, Matara, Sri Lanka

27: Also at Isfahan University of Technology, Isfahan, Iran

28: Also at Yazd University, Yazd, Iran

29: Also at Plasma Physics Research Center, Science and Research Branch, Islamic Azad University, Tehran, Iran

30: Also at Università degli Studi di Siena, Siena, Italy

31: Also at INFN Sezione di Milano-Bicocca; Università di Milano-Bicocca, Milano, Italy

32: Also at Purdue University, West Lafayette, USA

33: Also at International Islamic University of Malaysia, Kuala Lumpur, Malaysia

34: Also at Malaysian Nuclear Agency, MOSTI, Kajang, Malaysia

35: Also at Consejo Nacional de Ciencia y Tecnología, Mexico city, Mexico

36: Also at Warsaw University of Technology, Institute of Electronic Systems, Warsaw, Poland

37: Also at Institute for Nuclear Research, Moscow, Russia

38: Now at National Research Nuclear University 'Moscow Engineering Physics Institute' (MEPhI), Moscow, Russia 
39: Also at St. Petersburg State Polytechnical University, St. Petersburg, Russia

40: Also at University of Florida, Gainesville, USA

41: Also at P.N. Lebedev Physical Institute, Moscow, Russia

42: Also at California Institute of Technology, Pasadena, USA

43: Also at Budker Institute of Nuclear Physics, Novosibirsk, Russia

44: Also at Faculty of Physics, University of Belgrade, Belgrade, Serbia

45: Also at INFN Sezione di Roma; Sapienza Università di Roma, Rome, Italy

46: Also at University of Belgrade, Faculty of Physics and Vinca Institute of Nuclear Sciences, Belgrade, Serbia

47: Also at Scuola Normale e Sezione dell'INFN, Pisa, Italy

48: Also at National and Kapodistrian University of Athens, Athens, Greece

49: Also at Riga Technical University, Riga, Latvia

50: Also at Universität Zürich, Zurich, Switzerland

51: Also at Stefan Meyer Institute for Subatomic Physics (SMI), Vienna, Austria

52: Also at Istanbul University, Faculty of Science, Istanbul, Turkey

53: Also at Adiyaman University, Adiyaman, Turkey

54: Also at Istanbul Aydin University, Istanbul, Turkey

55: Also at Mersin University, Mersin, Turkey

56: Also at Cag University, Mersin, Turkey

57: Also at Piri Reis University, Istanbul, Turkey

58: Also at Izmir Institute of Technology, Izmir, Turkey

59: Also at Necmettin Erbakan University, Konya, Turkey

60: Also at Marmara University, Istanbul, Turkey

61: Also at Kafkas University, Kars, Turkey

62: Also at Istanbul Bilgi University, Istanbul, Turkey

63: Also at Rutherford Appleton Laboratory, Didcot, United Kingdom

64: Also at School of Physics and Astronomy, University of Southampton, Southampton, United Kingdom

65: Also at Instituto de Astrofísica de Canarias, La Laguna, Spain

66: Also at Utah Valley University, Orem, USA

67: Also at BEYKENT UNIVERSITY, Istanbul, Turkey

68: Also at Bingol University, Bingol, Turkey

69: Also at Erzincan University, Erzincan, Turkey

70: Also at Sinop University, Sinop, Turkey

71: Also at Mimar Sinan University, Istanbul, Istanbul, Turkey

72: Also at Texas A\&M University at Qatar, Doha, Qatar

73: Also at Kyungpook National University, Daegu, Korea 